Check for updates

Cite this: Phys. Chem. Chem. Phys., 2017, 19, 30261

Received 30th July 2017 ,

Accepted 18th October 2017

DOI: $10.1039 / c 7 c p 05155 \mathrm{~g}$

rsc.li/pccp

\section{Effect of donor to acceptor ratio on electrochemical and spectroscopic properties of oligoalkylthiophene 1,3,4-oxadiazole derivatives $\dagger$}

\author{
Aleksandra Kurowska, ${ }^{a}$ Pawel Zassowski, ${ }^{a}$ Anastasia S. Kostyuchenko, (D) bc Tatyana \\ Yu. Zheleznova, $\mathbb{D}^{\mathrm{b}}$ Kseniya V. Andryukhova, ${ }^{\mathrm{d}}$ Alexander S. Fisyuk, (D) ${ }^{\text {bd }}$ Adam Pron $^{\mathrm{e}}$ \\ and Wojciech Domagala (D) *a
}

\begin{abstract}
A structure-property study across a series of donor-acceptor-donor structures composed of mono- and bi-(1,3,4-oxadiazole) units symmetrically substituted with alkyl functionalized bi-, ter- and quaterthiophene segments is presented. Synthetically tailoring the ratio of electron-withdrawing 1,3,4-oxadiazole to electronreleasing thiophene units and their alkyl grafting pattern permitted us to scrutinize the impact of these structural factors on the redox, absorptive and emissive properties of these push-pull molecules. Contrasting trends of redox potentials were observed, with the oxidation potential closely following the donor-toacceptor ratio, whereas the reduction potential being tuned independently by either the number of acceptor units or the conjugation length of the donor-acceptor system. Increasing the thiophene unit contribution delivered a shift from blue to green luminescence, while the structural rigidity afforded by intramolecular non-covalent interactions between 1,3,4-oxadiazole and the thiophene moieties has been identified as the prime factor determining the emission efficiency of these molecules. All six structures investigated electropolymerize easily, yielding electroactive and electrochromic polymers. The polymer doping process is largely influenced by the length of the oligothiophene repeating unit and the alkyl chain grafting density. Polymers with relatively short oligothiophene segments are able to support polarons and polaron-pairs, whereas those with segments longer than six thiophene units could also stabilize diamagnetic charge carries - bipolarons. Increasing the alkyl chain grafting density improved the reversibility and broadened the working potential window of the $\mathrm{p}$-doping process. Stable radical anions have also been investigated, bringing detailed information about the conjugation pattern of these electron-surplus species. This study delivers interesting clues towards the conscious structural design of bespoke frontier energy level oligothiophene functional materials and their polymers by incorporating a structurally matching 1,3,4-oxadiazole unit.
\end{abstract}


releasing and electron withdrawing units. ${ }^{25,26}$ It has been demonstrated that the appropriate selection of building blocks with different Ionization Potential and Electron Affinities could give materials with bespoke properties for a particular application. ${ }^{1,25,27-30}$ In this building block approach, donoracceptor-donor (D-A-D) $\pi$-conjugated compounds featuring thiophene combined with heterocyclic 3,4-diazole derivatives have recently attracted particular attention. ${ }^{28,31,32}$

Thiophene is a popular organic semiconducting building block owing to its favourable arrangement of frontier molecular energy levels which can be modified through the derivatization of this heterocycle. ${ }^{33}$ Simple thiophene is considered a mild electron donor but this property can be tailored effectively by introducing different substituents at its carbon positions. ${ }^{29}$ Attaching long alkyl side chains, for example, improves the electron-releasing character as well as solubility. ${ }^{34,35}$ Satisfactory results in terms of the modulation of energy levels can be achieved by coupling thiophene segments with $\pi$-conjugated heterocycles characterized by a more electrophilic character. ${ }^{24,36}$ In particular, 1,3,4-oxadiazole distinguishes itself as a promising candidate due to its high photoluminescence quantum yield, hydrolytic and thermal stability as well as resistance to oxidative degradation. ${ }^{9}$ 1,3,4-Oxadiazole derivatives are frequently employed in organic optoelectronics, ${ }^{37}$ being used as emissive layers in OLEDs, ${ }^{9}$ electron injecting and transporting layers, ${ }^{8,20}$ hole blocking materials, ${ }^{9}$ or as fluorescent brightening agents. ${ }^{37}$

Several structural features make 1,3,4-oxadiazole particularly compatible with thiophene. First of all, both structures are fivemembered aromatic heterocycles where the 2,5-substitution pattern is the most $\pi$-conjugation efficient. Secondly, the absence of a hydrogen atom or other pendant group at the oxygen and nitrogen atoms eliminates potential steric hindrance problems with 2,5-substituted neighbours. The crystal structure and quantum chemical calculations show an almost zero dihedral angle between 1,3,4-oxadiazole and 2,5-substituted thiophene units, translating into an effective flattening of the conjugated system and improvement of local $\pi$-conjugation. ${ }^{38}$ Both the electron-withdrawing character of 1,3,4-oxadiazole and its efficient conjugation with thiophene afford versatile tuning of electronic properties of thiophene based molecular structures. ${ }^{36,39}$ Furthermore, oxygen and nitrogen atoms can engage in non-covalent interactions with sulphur and hydrogen atoms of adjacent thiophene units. ${ }^{38}$ In the anti-conformation, one can expect the formation of hydrogen bonds between oxadiazole's oxygen atom and the two hydrogen atoms of the flanking thiophenes, ${ }^{40}$ while each of the two symmetric azole nitrogens could couple with the nearest thiophene's sulphur atom by way of cooperative heteroatomic interactions. ${ }^{41}$ Each of these interactions contributes to stiffening and flattening of this structural motif, promoting the formation of well-organized molecular layers. One area where such properties are in demand is in Organic Field Effect Transistors. ${ }^{1,41}$ Taking advantage of thiophene's electropolymerization ability, bithiophene substituted oxadiazoles can be converted into regular alternating copolymers of ambipolar character, featuring stable $\mathrm{p}$ - and n-doped states. ${ }^{28,42}$ Redox transformations of these polymers produce reversible colouration changes of their films, giving efficient and controllable materials for ElectroChromic Devices. ${ }^{28,36}$

In this paper we present a systematic study of the redox and spectroscopic properties of donor-acceptor-donor (D-A-D) $\pi$-conjugated 1,3,4-oxadiazole-thiophene derivatives and their electropolymerization products. Intending to study the trends of changing the contributions of both donor and acceptor components, two structural variables have been investigated. The first one involved expanding the basic alkylbithiophene donor (D) motif by appending additional thiophene or alkylthiophene substituents to it, while the second one focused on the size and effectiveness of $\pi$-electron communication in the acceptor (A) core by introducing a second 1,3,4-oxadiazole unit either bound directly, or through a 1,4-phenylene spacer. This strategy afforded a precise tuning of donor-acceptor (D-A) interactions across a series of welldefined molecules of varying donor/acceptor ratio, as pictured in Fig. 1. A systematic study of these tailor-made molecules encompassing detailed spectroscopic, electrochemical and spectroelectrochemical characteristics is presented. The experimental findings are confronted with the results of quantum chemical (DFT) calculations delivering a mechanistic interpretation of the trends observed.

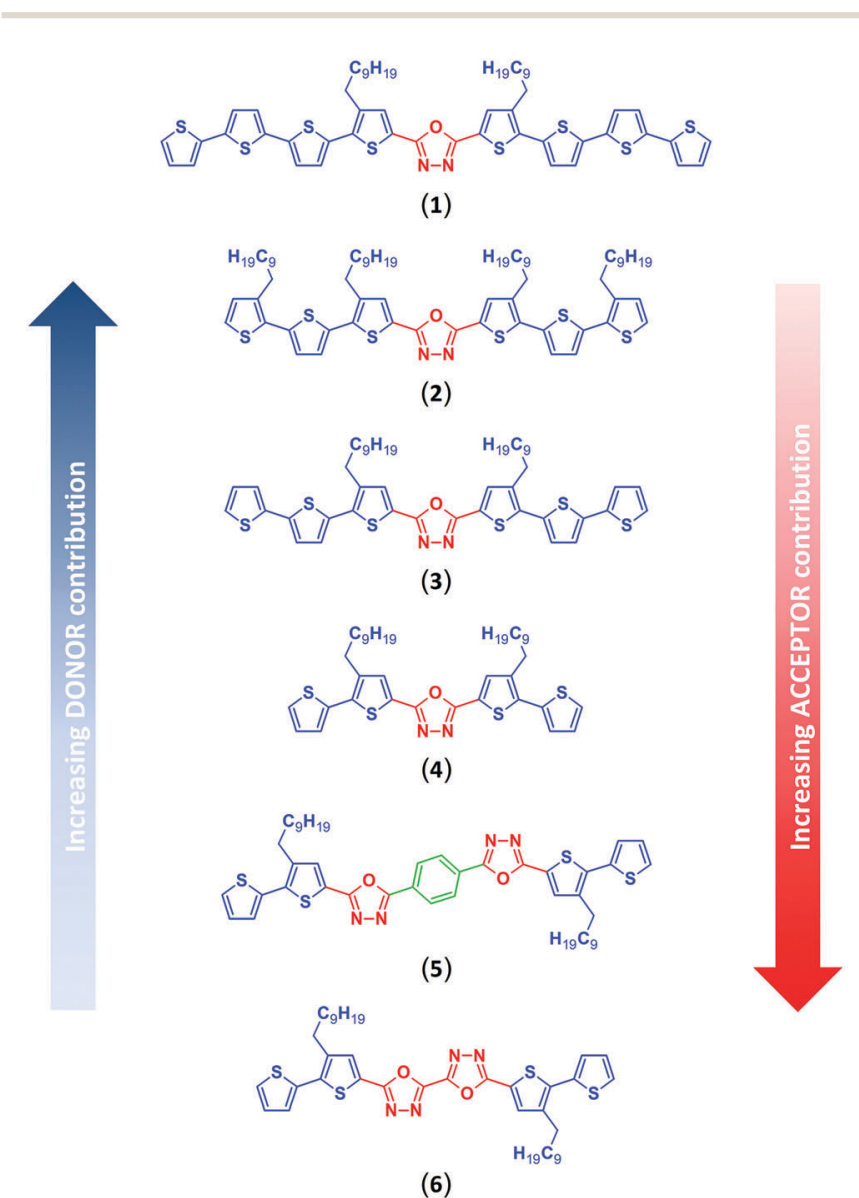

Fig. 1 Bespoke donor-to-acceptor ratio structures investigated in this study. 


\section{Experimental section}

\subsection{Synthesis}

Ester precursors (compounds 7a-c) of $\pi$-conjugated 1,3,4oxadiazole-thiophene derivatives $(\mathbf{1}, \mathbf{3}-\mathbf{6})$ were prepared by the method previously elaborated, ${ }^{43-47}$ starting from thiophene, 2,2'-bithiophene and 2,2':5', $2^{\prime \prime}$-terthiophene (Scheme 1).

Ethyl 3,3'-didecyl-[2,2':5', $2^{\prime \prime}$-terthiophen]-5-carboxylate (7d), the precursor of compound (2), was synthesized in a multi-step sequence starting from the bithiophene derivative (7a). ${ }^{43}$ The synthetic strategy involved the acetylation of compound (7a) with lauric acid chloride in the presence of $\mathrm{SnCl}_{4}$, followed by hydrolysis of the ester group, and finally decarboxylation using copper powder in isoquinoline. 1-(3'-Decyl-2,2'-bithien-5-yl) dodecane-1-one (10) was converted into the $Z$-/E-isomer mixture $(19 / 81)(11)$ by heating with DMF and $\mathrm{POCl}_{3}$. The reaction of this isomer mixture with thioglycolic acid ester in the presence of sodium ethoxide led to ethyl 3,3'-didecyl-[2,2': $5^{\prime}, 2^{\prime \prime}$-terthiophene]5-carboxylate (7d) (Scheme 2).

Heating the esters (7a-d) in an alcoholic solution of $\mathrm{KOH}$ or hydrazine hydrate led to carboxylic acids (12a-d) or hydrazides $(\mathbf{1 3 a}, \mathbf{b}, \mathbf{d})$, respectively (Scheme 3$).{ }^{43-47}$

Diacylhydrazines $(\mathbf{1 4 a}, \mathbf{b}, \mathbf{d})$, being direct precursors of the target 1,3,4-oxadiazole derivatives, were prepared by reacting compounds $(\mathbf{1 2 a}, \mathbf{b}, \mathbf{d})$ and $(\mathbf{1 3 a}, \mathbf{b}, \mathbf{d})$ in the presence of $N, N^{\prime}$-dicyclohexylcarbodiimide (DCC). Compound (14c) was synthesized in the reaction of hydrazine dihydrochloride in the presence of pyridine with the acid chloride, which was generated in situ from the carboxylic acid (12) by the action of chlorooxalyl chloride. Compound (15), in turn, was prepared from hydrazide (13a) and oxalyl chloride (Scheme 4). ${ }^{39}$

Heating diacylhydrazines (14a-d, 15) with phosphorus oxychloride led to formation of the desired bis(oligoalkylthiophene) derivatives of 1,3,4-oxadiazole (1-4, 6). After purification, the yields of the products $(2,3,4,6)$ were $78-94 \%$, while compound (1) was obtained in $10 \%$ yield only. Hightemperature acidic conditions of the oxadiazole ring closure step (Scheme 4) make thiophene rings susceptible to acid driven oligo/polymerization, ${ }^{48}$ consuming both substrates and products of the dehydration reaction of diacylhydrazines, and accounting for the observed decreasing yield of progressively longer oligothiophene derivatives of 1,3,4-oxadiazole, starting from 4 and 6 , through 2 and 3 , down to 1 .

The phenylene bisected bisoxadiazole derivative - 1,4-bis(5(3-decyl-[2,2'-bithiophen]-5-yl)-1,3,4-oxadiazol-2-yl)benzene (5) was obtained by heating 2,2'-bithiophene-5-carboxylic acid (7a) and 1,4-di(2H-tetrazol-5-yl)benzene in THF, in the presence of DCC (Scheme 5). The yield of compound (5) was $46 \%$.

Compounds (3), (4) and (6) have been synthesized according to procedures presented in our previous published works. ${ }^{39,43-47}$ A detailed synthetic protocol towards compounds $(\mathbf{1}, \mathbf{2}, \mathbf{5})$ is given in the ESI. $\dagger$

\subsection{Spectroscopy}

Absorption spectra were registered in tetrahydrofuran solutions using a PerkinElmer Lambda 750 spectrometer, whereas luminescence spectra were recorded on Agilent Cary Eclipse fluorescence spectrophotometer. The energy band gap $\left(E_{\mathrm{g}}\right)$ of compounds investigated was estimated from the onset of their $\pi-\pi^{*}$ absorption band. The photoluminescence quantum yield of 1,3,4-oxadiazole derivatives was estimated by comparison with a quantum yield standard: 9,10-diphenylanthracene $\left(Q_{\mathrm{R}}=0.95\right.$ when excited at 330-380 nm) or perylene $\left(Q_{\mathrm{R}}=0.93\right.$ when excited at $365-435 \mathrm{~nm})^{49}$ dissolved in ethanol, using a multi-point comparative method ${ }^{51}$ originating from the computational concept of Weber and Teale, ${ }^{50}$ and further developed by Dawson and Windsor. ${ }^{49}$ The proper excitation wavelength was chosen as described previously. ${ }^{36,39,44}$

\subsection{Electrochemistry}

All electrochemical measurements were performed in a $0.1 \mathrm{M}$ $\mathrm{Bu}_{4} \mathrm{NPF}_{6}$ (TCI Europe) electrolyte solution in dichloromethane (Sigma-Aldrich, Chromasolv for HPLC) for the anodic potential range, or in anhydrous tetrahydrofuran (Acros Organics) for the cathodic potential range, using a MultiAutolab M101 potentiostat. All the solutions were purged with argon before the measurements. Experiments were performed in an electrochemical cell fitted with a platinum disk working electrode, platinum coil as the counter electrode and silver wire as a pseudo-reference electrode. The potential of the silver pseudo-reference electrode was calibrated versus the ferrocene redox couple, measured in the same electrolyte solution.

Electropolymerization of the compounds studied was performed using cyclic voltammetry from a $1 \mathrm{mM}$ (for compounds 2 to 6 ) or $0.25 \mathrm{mM}$ (for compound 1) monomer and a $0.1 \mathrm{M} \mathrm{Bu}_{4} \mathrm{NPF}_{6}$ electrolyte solution in dichloromethane, at a $100 \mathrm{mV} \mathrm{s}^{-1}$ scan rate. After deposition, the polymer film on the electrode was washed with pure solvent and transferred into a monomer-free electrolyte solution for further investigations. Redox cycling was performed in the same electrolyte environment as that used for electropolymerization.

\subsection{UV-Vis-NIR and EPR spectroelectrochemistry}

The generation of radical ions (or diions) upon electrochemical oxidation or reduction of solutions of the compounds investigated was carried out and probed in situ using spectroelectrochemical methods. Spectral measurements were done using a UV-Vis Hewlett Packard 8453 spectrophotometer and a JEOL JES FA-200 X-band continuous wave Electron Paramagnetic Resonance (EPR) spectrometer operating at a $100 \mathrm{kHz}$ field modulation. Individual spectrometers were coupled with an OMNI 90 potentiostat in case of UV-Vis spectroelectrochemical measurements or an Autolab PGSTAT 100N potentiostat for EPR investigations. For these experiments, the concentration of a given compound and measurement conditions were identical to those used in the electrochemical studies. The spectroelectrochemical experiments were performed in custom-made cells. For UV-Vis spectroelectrochemistry, the cell comprised a quartz $10 \mathrm{~mm}$ UV-Vis cuvette fitted with an ITO coated glass slide as a working electrode pressed against the inner side of the cuvette window using a Teflon wedge sunk at the bottom of the cuvette in the working solution pool where the counter and reference 


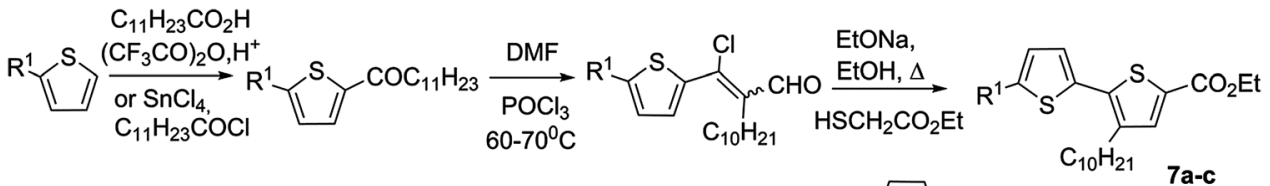

$$
\begin{aligned}
& \begin{array}{lll}
\text { a. } \mathrm{R}_{1}=\mathrm{H} & \text { b. } \mathrm{R}_{1}=\left\langle\mathrm{R}_{\mathrm{S}}\right. & \text { c. } \mathrm{R}_{1}=4
\end{array}
\end{aligned}
$$

Scheme 1 Synthesis of ester precursors $(7 a-c)$.
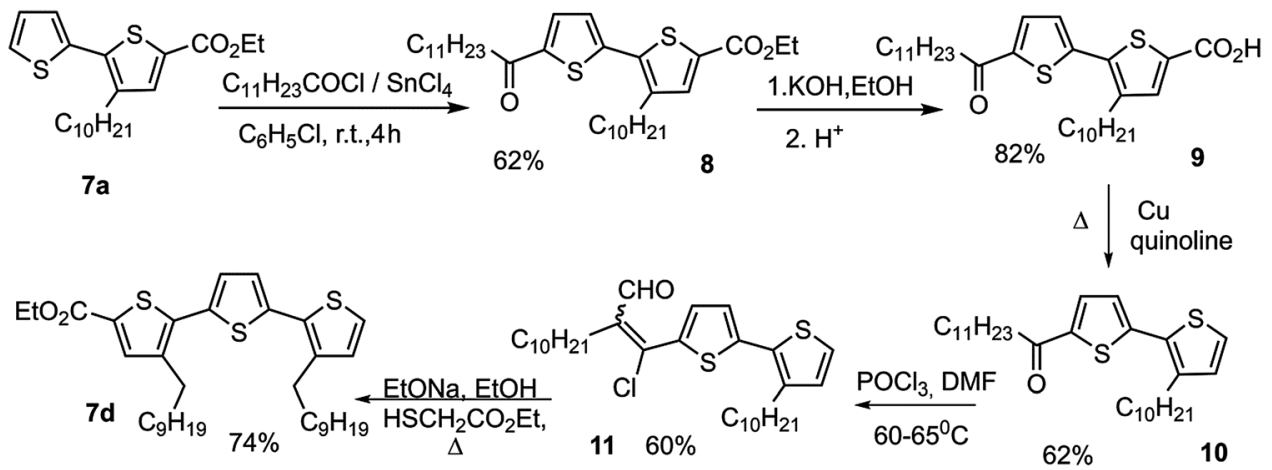

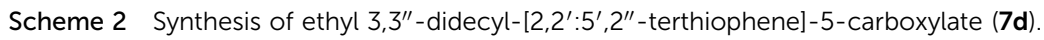

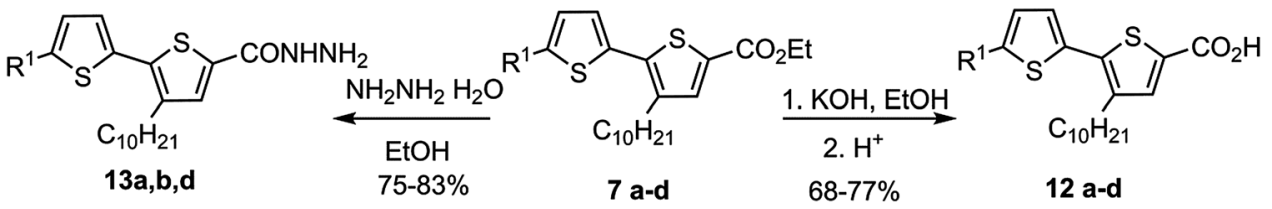

Scheme 3 Synthesis of acids (12a-d) and carbohydrazide (13a, b, d).

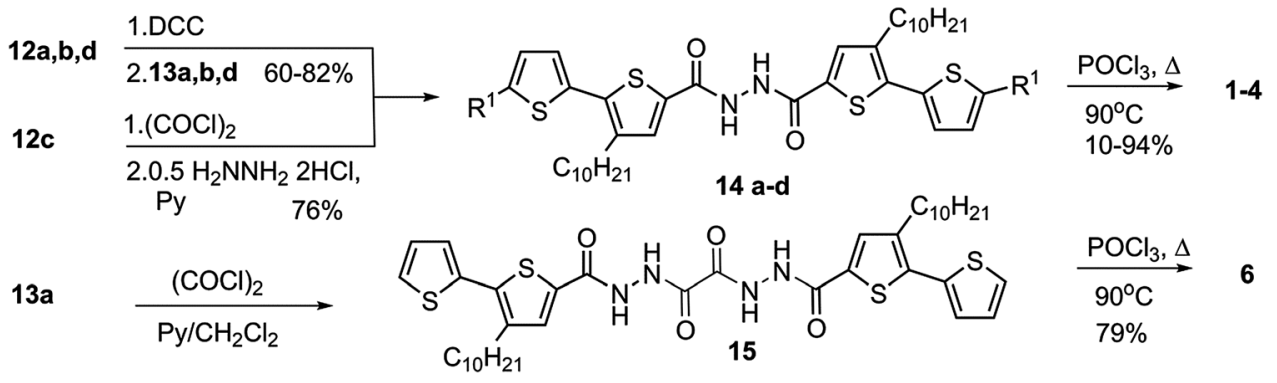

Scheme 4 Synthesis of oligothiophene derivatives of 1,3,4-oxadiazole $(\mathbf{1 - 4}, \mathbf{6})$.

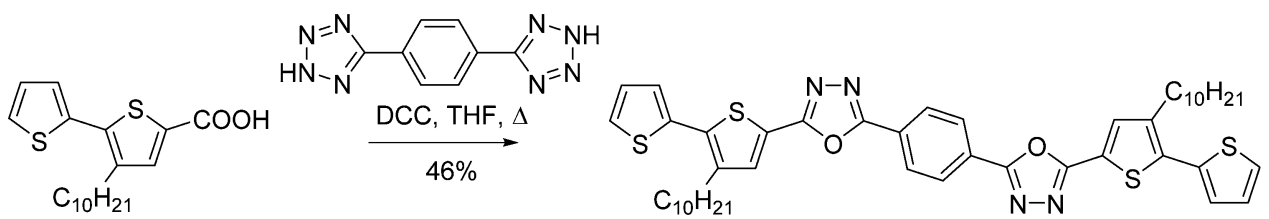

Scheme 5 Synthesis of alkylbithiophene derivative of 1,4-bis-(1,3,4-oxadiazol-2-yl)benzene (5).

electrodes were immersed. In order to secure a reproducibly thin $(100 \mu \mathrm{m})$ layer of the solution studied, a Teflon gasket was placed between ITO electrode and the wall of the quartz cuvette used. EPR spectroelectrochemistry was conducted in a thin cylindrical glass tube narrowed at the bottom where platinum wire working electrode was inserted. In both assemblies, platinum coil was used as an auxiliary electrode and a silver electrode as a quasireference electrode. Redox reactions were triggered by applying 
an appropriate potential and maintaining it throughout the measurement. Microwave power and modulation amplitude were adjusted to accurately record hyperfine structured EPR spectra of the generated radicals. EPR line shape was analysed using WinSim software ${ }^{52}$ by optimizing crude hyperfine coupling constants estimated from computationally derived spin densities at individual atoms of the radical ions.

A different experimental setup was used for investigating polymer layers. Their UV-Vis-NIR spectroelectrochemical measurements were carried out using paired Ocean Optics QE65000 and NIRQuest512 spectrometers. Polymers were deposited on an ITO coated glass electrode under the same analytical conditions as those used during the electropolymerization experiments. The spectroelectrochemical cell comprised a $2 \mathrm{~mm}$ quartz cuvette, with polymer coated ITO as the working electrode, platinum mesh as the counter electrode and Teflon clad thin silver wire as the reference electrode. A thin, tuning fork shaped Teflon gasket separated the working and the counter electrode. UV-Vis-NIR spectra were taken in the potentiostatic mode at stepwise incremented potentials applied to the polymer film.

For EPR spectroelectrochemistry, polymer films were deposited on a platinum wire electrode, again using the same conditions as used during the electrochemical experiments. Apart from differences in sample preparation, the experimental setup was identical to that used to study the radical anions of the compounds investigated in solution. Spectra were taken in potentiostatic mode, first during oxidative doping (incrementing potentials) and then during the subsequent de-doping (decrementing potentials) of the polymer film. Double integration of the recorded first derivative EPR spectra gave the relative number of paramagnetic centres, and the abscissa axis distance between their up and down peaks was taken as the signal linewidth $\left(\Delta B_{\mathrm{pp}}\right)$.

\subsection{DFT calculations}

DFT/TDDFT calculations were carried out with the B3LYP ${ }^{53-55}$ hybrid functional combined with the 6-31G(d) basis set. Ground state geometries of the compounds investigated (with alkyl chains shortened to ethyl groups) were optimized to a local minimum with no symmetry constraints, followed by frequency calculations. In all the cases, no imaginary frequencies were found. TDDFT calculations were performed as follows: first, the excited state geometry was simulated at the B3LYP/6-31G(d) level of theory, while for optical transitions of the ground state, the functional was changed to CAM-B3LYP. ${ }^{56}$ All the calculations in this work were conducted with the polarizable continuum model (PCM) using dichloromethane or tetrahydrofuran as the solvent, as implemented in Gaussian 09 software. Input files and molecular orbital plots were prepared with Gabedit 2.4.7 software. ${ }^{57}$ All the calculations were carried with Gaussian 09 software. ${ }^{58}$

\section{Results and discussion}

\subsection{Spectroscopic properties}

The absorption spectra of each investigated compound (Fig. 2a) show a single, featureless band with a single maximum $\left(\lambda_{\max }\right)$
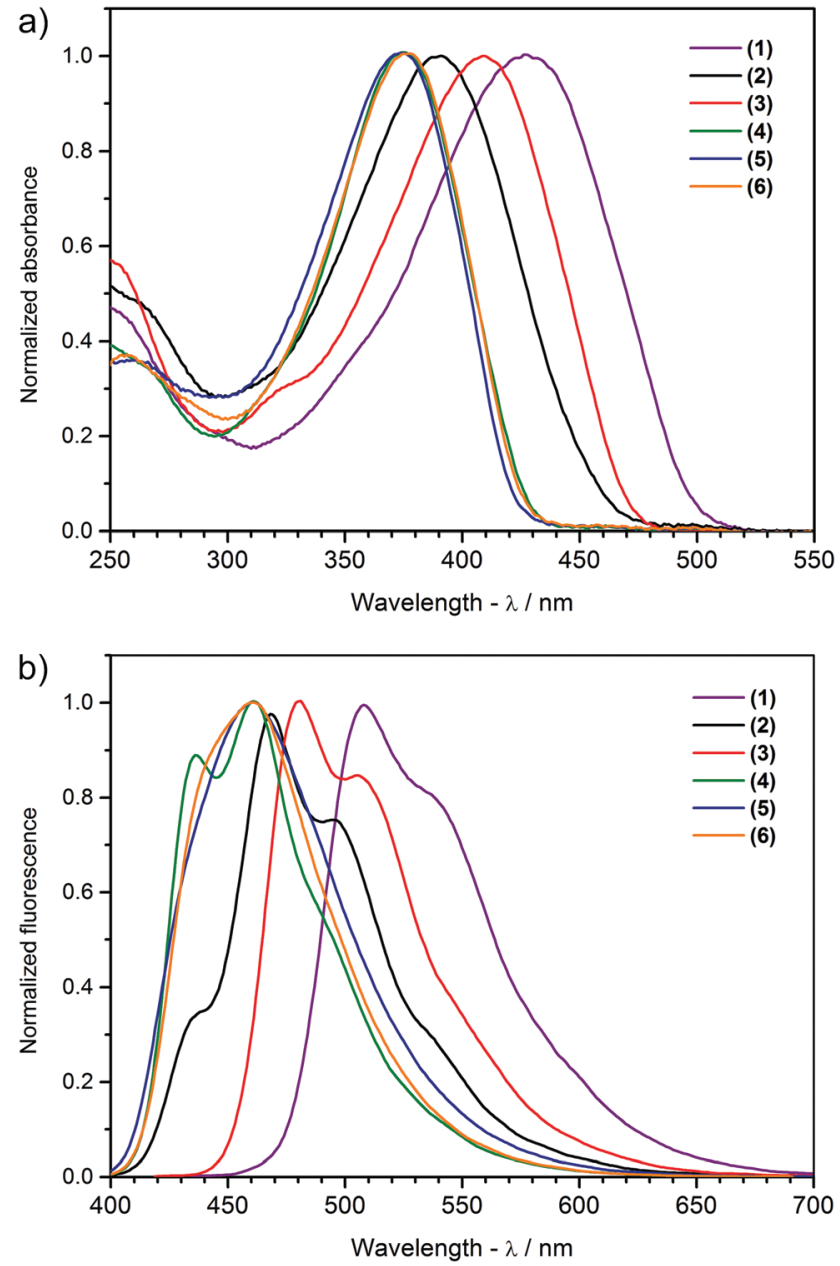

Fig. 2 Normalized (a) absorption and (b) luminescence spectra of the compounds investigated in tetrahydrofuran solution.

Table 1 Spectroscopic properties of the compounds studied dissolved in tetrahydrofuran

\begin{tabular}{|c|c|c|c|c|c|c|}
\hline \multirow[b]{2}{*}{ Compound } & \multicolumn{3}{|c|}{ UV-Vis } & \multicolumn{3}{|c|}{ Photoluminescence } \\
\hline & $\begin{array}{l}\lambda_{\max }{ }^{a} \\
{[\mathrm{~nm}]}\end{array}$ & $\begin{array}{l}E_{\mathrm{g}}^{\mathrm{opt}} \\
{[\mathrm{eV}]}\end{array}$ & $\begin{array}{l}\lambda_{\max }{ }^{b} \\
{[\mathrm{~nm}]}\end{array}$ & $\begin{array}{l}\lambda_{\mathrm{em}}{ }^{c} \\
{[\mathrm{~nm}]}\end{array}$ & $\begin{array}{l}\Delta^{d} \\
{[\mathrm{eV}]}\end{array}$ & $\Phi_{\mathrm{fl}}{ }^{e}$ \\
\hline 1) & 427 & 2.49 & 411 & $508 ; 537$ & 0.46 & $0.18 \pm 0.01$ \\
\hline 2) & 391 & 2.73 & 375 & $436 ; 468 ; 496$ & 0.52 & $0.22 \pm 0.01$ \\
\hline 3) & 410 & 2.64 & 391 & $480 ; 506$ & 0.44 & $0.17 \pm 0.01$ \\
\hline 4) & 376 & 2.92 & 342 & $434 ; 461$ & 0.61 & $0.88 \pm 0.04$ \\
\hline 5) & 375 & 2.95 & 353 & 460 & 0.61 & $0.60 \pm 0.02$ \\
\hline (6) & 378 & 2.92 & 360 & $441 ; 460$ & 0.58 & $0.63 \pm 0.03$ \\
\hline
\end{tabular}

${ }^{a}$ Absorption maxima. ${ }^{b}$ Simulated with CAM-B3LYP/6-31G(d)/PCM(THF). ${ }^{c}$ Emission band maxima. ${ }^{d}$ Difference between absorption and emission maximum. ${ }^{e}$ Quantum efficiency with experimental error.

positioned between 375 and $427 \mathrm{~nm}$ (Table 1), shifting bathochromically with increasing number of thiophene units (compare (1), (3) and (4) in Fig. 2a). The greater the number of thienylene units in conjugation, the better the delocalization of the $\pi$-electron density along the main chain, translating into a diminishing energy gap between the HOMO and LUMO levels. An additional alkyl chain at the $\beta$-position of the terminal thiophene ring widens 
the band gap in (2) by $0.09 \mathrm{eV}$ compared to (3) by elevating the ground state torsional angle within the terminal alkylbithiophene groups of (2). Surprisingly, there appears to be little to no effect of the number, or spacing pattern, of the 1,3,4-oxadiazole units on the peak maxima of the absorption bands of compounds (4), (5) and (6), with differences amounting to $2 \mathrm{~nm}$ only, suggesting an almost identical energy displacement of both the HOMO and LUMO levels with increasing size of the acceptor core. Both the above described trends are clearly reflected in the results of TD DFT calculations. At the first attempt, optical transitions were simulated with the same functional and basis set as the one used for geometry optimization (Table S1, ESI $\dagger$ ); however, computed excitation energies were hugely red-shifted compared to the experimental spectra. Improved values, close to experimental, were obtained when the functional was changed to CAM-B3LYP (Table S2, ESI $\dagger$ ). Apart from the different excitation energies obtained, both functionals led to similar conclusions: the absorption feature pictured in Fig. 2a is principally associated with a HOMO to LUMO $\left(\pi-\pi^{*}\right)$ transition, while the other transitions, having much smaller oscillator strengths, position themselves well into in the UV region, which is typical for oligothiophenes. ${ }^{59}$

The investigated 1,3,4-oxadiazole derivatives are photoluminescent, emitting blue to green light in tetrahydrofuran solution, with emission maxima in the range of 434 to $537 \mathrm{~nm}$ (Table 1 and Fig. 2b). This property is preserved in the solid state when these derivatives are dispersed in an amorphous matrix, as has been demonstrated for (4) and (6) in prototype host-guest OLED devices. ${ }^{39}$ Contrary to the featureless $\pi-\pi^{*}$ absorption bands, the emission spectra of (1), (2), (3) and (4) show a clear vibronic structure with at least two well discernible components (Fig. 2b and Table 1). Vibronic coupling points to a rigid geometry of the excited state, which is confirmed by DFT calculations predicting an almost ideally planar geometry of all the molecules in their first singlet excited state (Table S3, ESI $\dagger) .{ }^{60}$ Emission and absorption maxima are displaced by 0.44-0.52 eV for compounds with additional electron-releasing thiophene units (1) to (3), and $0.58-0.61 \mathrm{eV}$ for molecules with a greater contribution of electron-withdrawing moieties (4) to (6) (Table 1), implying that a notable geometric reorganization takes place between the ground and excited states. ${ }^{61}$

The fluorescence quantum efficiency of $\pi$-conjugated molecules depends on the interplay of various structural factors determining the preferential excited state relaxation channels. For the compound series investigated, the quantum yield clearly changes with the number of donor and acceptor units in a molecule. Compounds (1) and (3) turn out to be the weakest emitters, demonstrating yields of 0.18 and 0.17 , respectively (Table 1). The quantum yield improves slightly when a second pair of alkyl chains is introduced in compound (2). A huge increase is observed for compound (4), where the oligothiophene arm is only two thiophene units long, with a quantum yield reaching 0.88 - the highest value of all the molecules examined. Curiously enough, doubling the number of 1,3,4-oxadiazole heterocycles results in a decrease, rather than increase, of fluorescence efficiency, with the quantum yields of compounds (5) and (6) being roughly three-quarters of that of compound (4).
Three possible contributing factors are brought to attention to address these experimental observations. The first is the number of thiophene groups in a molecule, the second is the overall length of its $\pi$-conjugated system, and the third is the structural flexibility of each molecule. The three structures featuring identical alkylbithiophene moieties, (4), (5) and (6), fluoresce distinctively better than compounds (1), (2) and (3) having ter and quaterthiophene arms. The sulphur atom is known to contribute to fluorescence quenching by way of the heavy atom effect, fostering competitive radiationless excited state decay modes. ${ }^{61,62}$ This effect appears to saturate quickly though, for compound (1) shares almost identical quantum yield values with compounds (2) and (3) despite having an extra two thiophene rings. Correlating the size of the conjugated double bond system with the quantum yield of a compound, one can trace an inverse relationship, with the shortest molecule (4) being the best fluorophore. The reason here could be the stability of photogenerated charged species, which improves with increasing conjugation length, giving time for intermolecular external conversion processes to take place. The effectiveness of $\pi$-conjugation depends primarily on the planarity of the atomic scaffold, which, in turn, depends on both the covalent and non-covalent interactions prevailing in a molecule. Computational optimization of the geometry of compounds (1), (2), (3), (4), indicates the bis $\left(4^{\prime}\right.$ decylthiophene)-1,3,4-oxadiazole motif as being the most rigid fragment of these molecules, remaining planar both in the ground and in the $\mathrm{S}_{1}$ excited state (Table S3, ESI $\dagger$ ). The reasons behind this are the hydrogen bonds between the oxygen and nearby hydrogen atoms of each of the two neighbouring thiophene units. Similar cooperative interactions are expected between the phenylene protons and oxygen and nitrogen atoms of the 1,3,4-oxadiazole units in compound (5), giving a plausible clue to its lower quantum yield due to an improved $\pi$-conjugation. Our previous study indicated that intramolecular rotation can be responsible for enhancing radiationless quenching of the excited state in this class of molecules. ${ }^{39}$ While a flat geometry is predicted for all six molecules in their excited state (Fig. 3), intermolecular interactions with the solvent molecules can activate energetically accessible rotational modes of individual molecular fragments. Looking at the contours of the LUMO orbital, one finds that in molecules (1), (2), (3) and (4) conjugation effectively ceases at the penultimate thiophene ring of each oligothiophene arm. The barriers to rotation of the terminal thiophene groups are hence minimized, making it possible to thermally depopulate the excited state of these molecules with comparable efficiency. A similar situation is observed in (5) and (6) where the terminal thiophene rings are out of conjugation too. This can explain the impaired quantum yield of these bioxadiazole derivatives with respect to compound (4), whose LUMO level appears to extend over the whole molecule. Considering the factors discussed above, the feasibility of intramolecular rotation of the terminal thiophene units explains the observed trends of fluorescence quantum yield in the most consistent manner. 
(1)

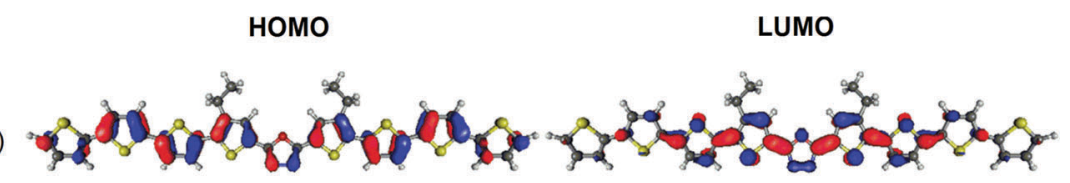

(2)
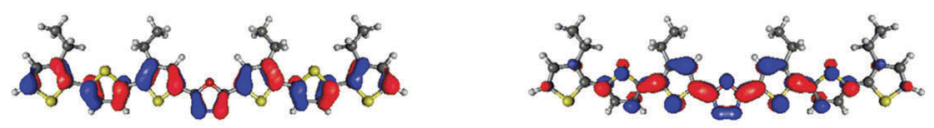

(3)
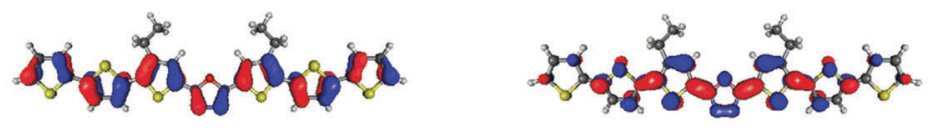

(4)
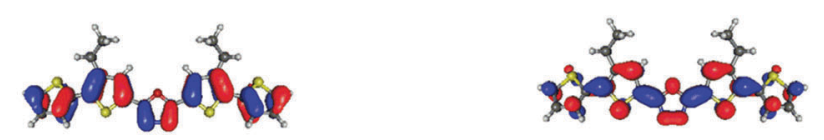

(5)
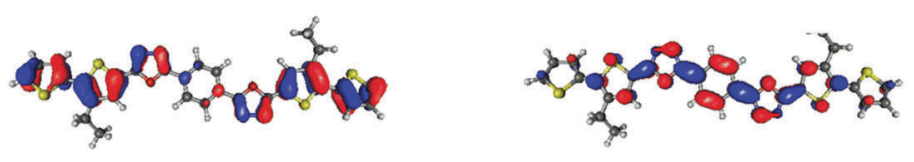

(6)
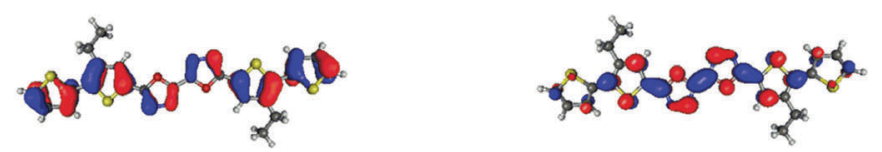

Fig. 3 Shape of the frontier orbitals of the molecules investigated, calculated at B3LYP/6-31G(d)/PCM(DCM or THF). The isovalue is equal to 0.03 e a.u..$^{-3}$ in each case.

\subsection{Electrochemistry of positively and negatively charged redox states}

Electrochemical measurements were conducted in order to determine the influence of the donor-acceptor balance on the redox properties of the six 1,3,4-oxadiazole derivatives investigated. For this purpose, the electrochemistry of compounds (1) to (6) was studied using cyclic voltammetry. Under certain circumstances, this technique can be considered an appropriate equivalent of more complex methods, such as UPS and IPES, ${ }^{63-66}$ allowing the determination of two crucial parameters of organic semiconductors: Ionization Potential (IP) and Electron Affinity (EA), ${ }^{67}$ which determine the working parameters of optoelectronic devices. ${ }^{3}$ Electrochemical studies reveal that all the investigated molecules are electroactive in both the anodic and cathodic potential range. In the case of (1) a quasi-reversible oxidation peak is observed (Fig. 4a and Fig. S1, ESI $\dagger$ ), with a characteristic sharp reduction peak after the reversal of the polarization direction. ${ }^{68}$ Similar features are observed in the electrochemistry of long oligothiophenes in the solid state. ${ }^{69-73}$ Here the reason is that the radical cation of (1) is insoluble and precipitates at the electrode. A subsequent sharp reduction peak is associated with reduction of the cation of (1) back to the neutral form, followed by its dissolution from the electrode surface. Contrary to (1), compounds (2) through to (6) demonstrate an irreversible oxidation peak originating from the formation of a radical cation capable of $\mathrm{C}_{\alpha}-\mathrm{C}_{\alpha}$ coupling, yielding an electroactive polymer deposit at the surface of the electrode (Fig. 4a and Fig. S2, ESI $\dagger$ ). Compound (1) also undergoes polymerization, albeit at potentials past its second oxidation peak (Fig. S2, ESI $\dagger$ ). These trends resemble those observed for short oligothiophenes, with bithiophene and terthiophene undergoing efficient polymerization at their first oxidation peak, and quaterthiophene demonstrating a well-defined reversible redox couple. The reactivity of the radical cation of progressively longer thiophene oligomers decreases exponentially, making the quinquethiophene radical cation stable. ${ }^{73,74}$ Quantum chemical computations predict uninterrupted $\pi$-conjugation of the HOMO orbital across four or more thiophene units in the compounds investigated (Fig. 3), suggesting that the electron-withdrawing nature of the 1,3,4-oxadiazole units is responsible for destabilizing, and hence intensifying, the reactivity of the radical cations. ${ }^{36,42}$ Equally likely, the 1,3,4-oxadiazole unit could hinder the delocalization of positive charge over itself, disrupting electron communication between oligothienyl arms and making them behave more or less independently, explaining the observed trends of the radical cation reactivity.

Analysis of the data listed in Table 2 leads to the conclusion that the oxidation potential, and thus IP, depends directly on the length of donor segment. With increasing number of thiophene units, the oxidation potential decreases from (1) to (4) with $\left|\Delta E_{1-4}\right|=0.40 \mathrm{~V}$. The number of alkyl substituents in the donor segment also affects the oxidation potential of the molecule. The additional alkyl chains in (2) decrease the oxidation potential ${ }^{75,76}$ by about $\left|\Delta E_{2-3}\right|=0.07 \mathrm{~V}$ compared to (3), 
a)

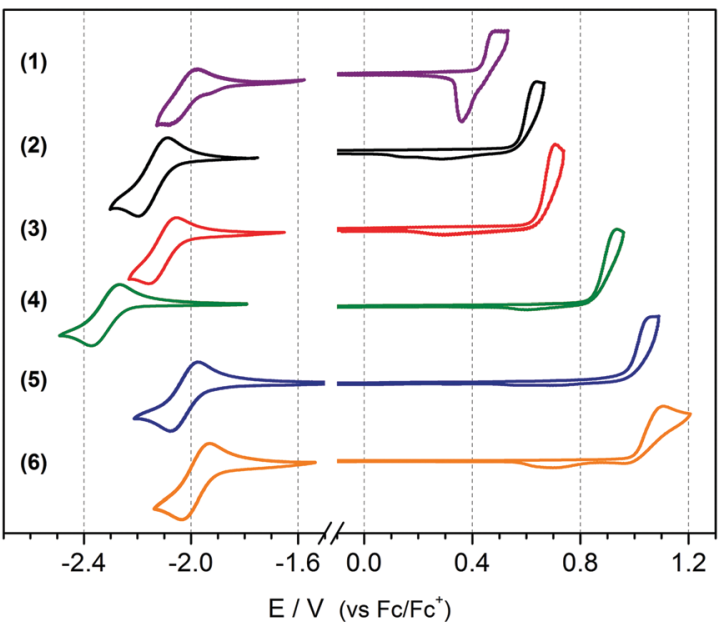

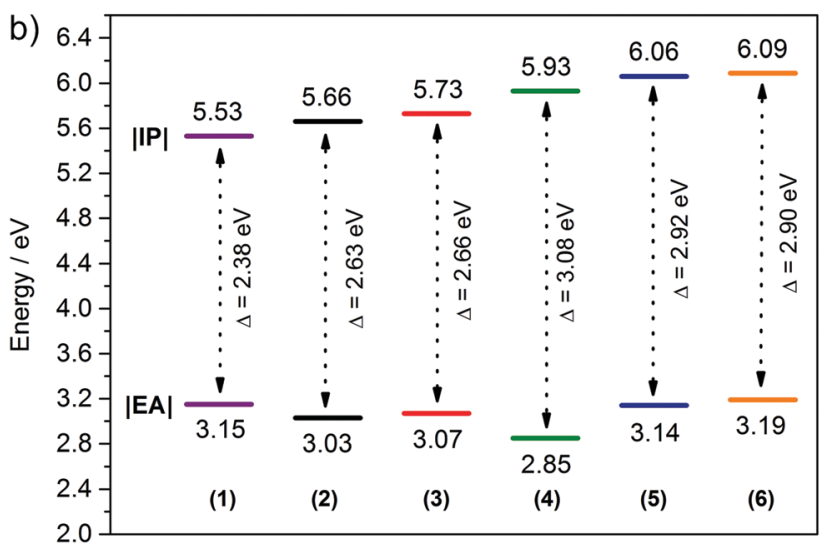

Fig. 4 (a) Cyclic voltammograms of the investigated monomers registered in $0.1 \mathrm{M} \mathrm{Bu}_{4} \mathrm{NPF}_{6}$ in dichloromethane for the anodic potential range, and in tetrahydrofuran for the cathodic potential range. Scan rate: $100 \mathrm{mV} \mathrm{s}^{-1}$. (b) absolute values of lonization Potential (IP) and Electron Affinity (EA) of the compounds investigated.

owing to the positive inductive effect of alkyl substituents. Conversely, doubling the number of 1,3,4-oxadiazole rings pushes the oxidation potential to higher values $\left|\Delta E_{4-6}\right|=0.16 \mathrm{~V}$. This comes about as a result of the electron-withdrawing properties of this heterocycle, lowering the $\pi$-electron density on the adjacent alkylbithiophene segment, and making the formation of the radical cation more difficult. ${ }^{36,42}$

All the molecules investigated demonstrate an ambipolar behaviour, undergoing both oxidation and reduction processes. Their first reduction step is characterized by a reversible redox couple (Fig. 4a) followed by a second, well-separated, quasireversible couple at lower potentials (Fig. S3, ESI $\dagger$ ). These results indicate that the radical anion of each compound is stable, whereas the addition of a second electron produces less stable dianion species. While oxidation potentials decrease with an increasing ratio of acceptor to donor units in a molecule, the reduction potentials appear to follow a less obvious trend. Doubling the number of oxadiazole groups in compounds (5) and (6) definitely shifts the reduction potential to less negative values compared to compound (4), consistent with the expected improved delocalization of the negative charge over two electron-withdrawing 1,3,4-oxadiazole units rather than over one. However, comparing compounds (1), (2), (3) and (4), all having a single 1,3,4-oxadiazole unit, it becomes clear that elongation of the oligothiophene segment can be just as effective in stabilizing the negatively charged redox state, as expanding the electron-withdrawing moiety is, since the onset reduction potential of (1) is just $40 \mathrm{mV}$ more negative than that of (6). Stable radical anions have been observed in oligothiophene systems, ${ }^{73,77}$ indicating that extended $\pi$-conjugated networks of these heterocycles can feature ambipolar properties. It is also interesting to look at the separation of the first and second cathodic peaks, corresponding to the generation of a radical anion and dianion in the molecules investigated. Going from (1) through to (6), this peak separation parameter grows with the decreasing donor-to-acceptor ratio, indicative of a changing delocalization pattern of the surplus electron over the $\pi$-conjugated scaffold. A diminishing peak separation between the first and second redox couple suggests a shortening of the conjugation length of the negatively charged species relative to the formal size of the $\pi$-conjugated system in a molecule, making it increasingly facile to introduce a second charge into the system. Spin pairing could also play a role here, since moderate-length oligothiophenes are known to favour electron-even charged states. ${ }^{70}$ Detailed investigation of the radical anion species will be presented further on.

The experimental measurements are supported by DFT calculations; the simulated energies of frontier orbitals and the energy gaps are collected in Table 2, while the shapes of the frontier orbitals are presented in Fig. 3. The calculated values of orbital energy follow those from the experiments, with errors typical for the B3LYP/6-31G(d) combination, ${ }^{78,79}$ predicting a reduction of the energy gap with the addition of electron donors and a shifting of the energy of both frontier orbitals down with the addition of electron acceptors. For all the molecules examined, except (5), the HOMO level is delocalized over the entire molecule, whereas the LUMO level tends to be present in the central part of a molecule, as discussed earlier. Different results were obtained for molecule (5), where the 1,4-phenylene linker expanding the acceptor core leads to a partial separation of frontier orbitals. Here the HOMO orbital is missing from the central phenyl ring, whereas the LUMO orbital is present almost entirely on the 1,4-bis(1,3,4-oxadiazol2-yl)phenylene acceptor moiety.

The long lifetime of the radical anions of the compounds studied permitted investigating their spectroscopic properties. UV-Vis spectra of the reduction process and EPR spectra of the products obtained are shown in Fig. 5 and Fig. S4 (ESI $\dagger$ ), whereas the associated spectroscopic data are collected in Table 3. Upon reduction, the intensity of the HOMO-LUMO transition band of the neutral compound decreases, accompanied by the appearance of a strong higher energy band in the UV region and a weak shoulder band in the visible part of spectrum. Usually, reduction of D-A-D type conjugated compounds results in the formation of new, strong bands ascribed to transitions typical for radical anions or dianions, ${ }^{80-83}$ yet recently we have shown that the reduction of some molecules 
Table 2 Electrochemical and electronic properties of compounds (1) to (6) determined from their redox potentials in dichloromethane for the anodic potential range and in tetrahydrofuran for the cathodic potential range

\begin{tabular}{|c|c|c|c|c|c|c|c|c|}
\hline Compound & $E_{\text {(onset) }}^{\text {ox }}[\mathrm{V}]$ & $E_{\text {(onset) }}^{\text {red }}[\mathrm{V}]$ & $|\mathrm{IP}|^{a}[\mathrm{eV}]$ & $|\mathrm{EA}|^{b}[\mathrm{eV}]$ & $E_{\mathrm{g}}^{\mathrm{el}}[\mathrm{eV}]$ & $\mathrm{HOMO}^{c}[\mathrm{eV}]$ & $\mathrm{LUMO}^{d}[\mathrm{eV}]$ & $E_{\mathrm{gDFT}}^{e}[\mathrm{eV}]$ \\
\hline (2) & +0.56 & -2.07 & 5.66 & 3.03 & 2.63 & -5.16 & -2.26 & 2.90 \\
\hline (5) & +0.96 & -1.96 & 6.06 & 3.14 & 2.92 & -5.58 & -2.39 & 3.19 \\
\hline (6) & +0.99 & -1.91 & 6.09 & 3.19 & 2.90 & -5.88 & -2.47 & 3.41 \\
\hline
\end{tabular}

${ }^{a}$ Calculated according to the equation IP $[\mathrm{eV}]=|e|\left(E_{\text {(onset) }}^{\text {ox }}+5.1\right) .{ }^{b}$ Calculated according to the equation EA $[\mathrm{eV}]=-|e|\left(E_{(\mathrm{onset})}^{\mathrm{red}}+5.1\right) .{ }^{c}$ Simulated with B3LYP/6-31G(d)/PCM(DCM). ${ }^{d}$ Simulated with B3LYP/6-31G(d)/PCM(THF). ${ }^{e}$ Calculated as a difference between simulated HOMO and LUMO energies.

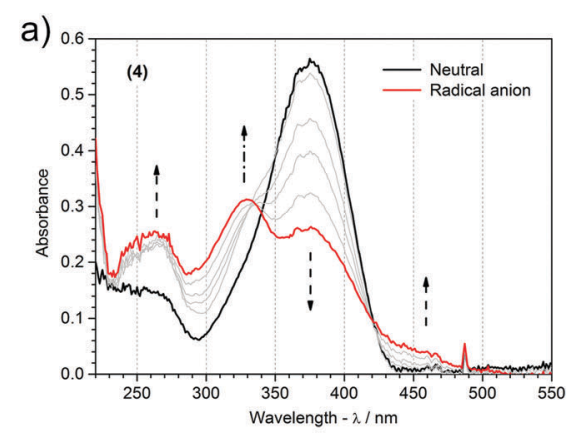

d)

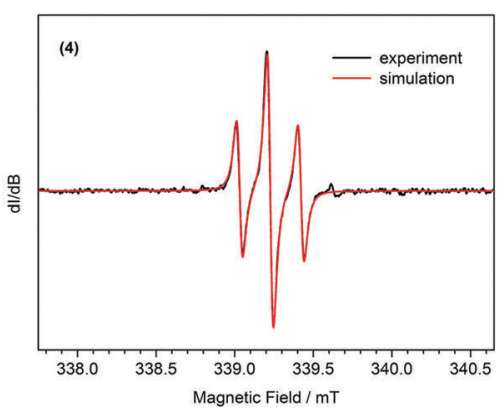

g)

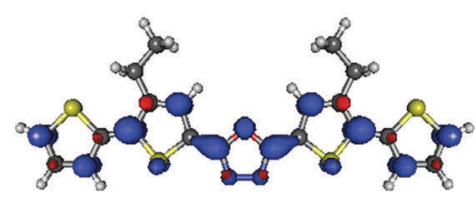

b)

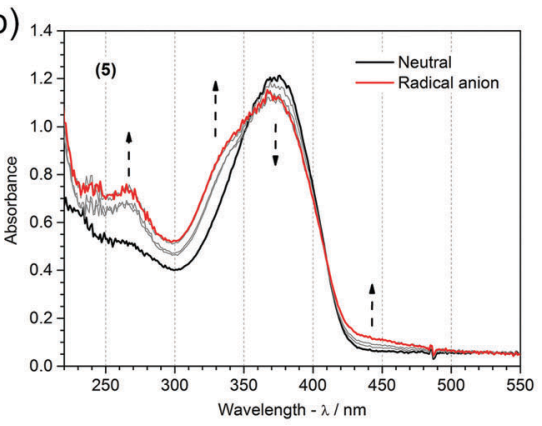

e)

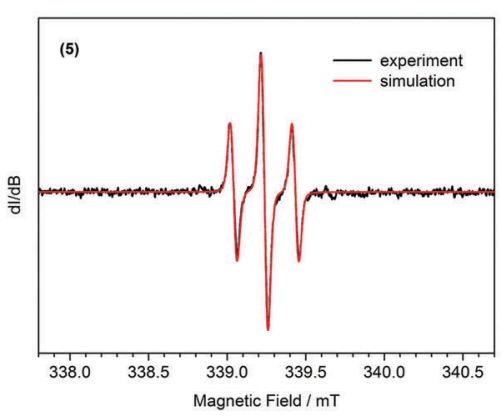

h)

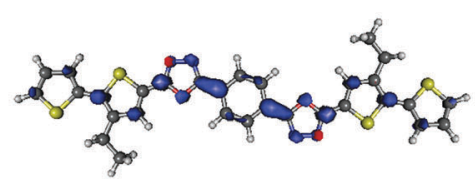

c)

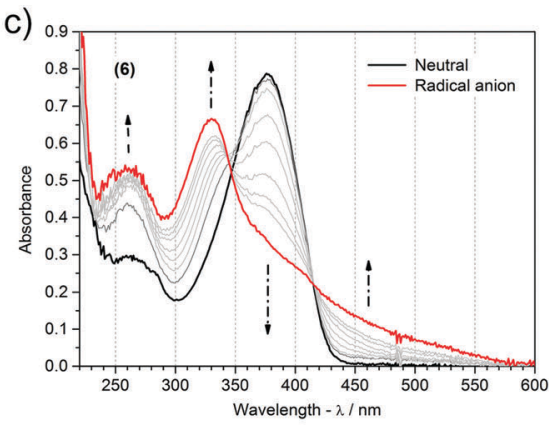

f)

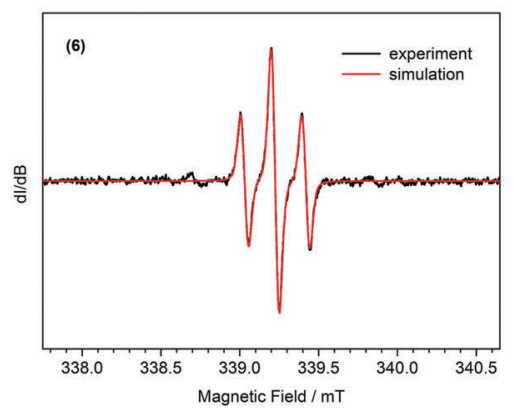

i)

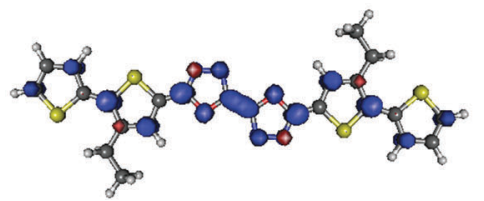

Fig. 5 In situ spectroelectrochemistry of compounds (4), (5) and (6). (a-c) UV-Vis spectra recorded during electrochemical reductions. (d-f) EPR spectra of the electrogenerated radical anions. (g-i) Spin densities of the radical anions calculated at uB3LYP/6-31G(d)/PCM(THF) with an isovalue equal to 0.005 e a.u. ${ }^{-3}$ in each case.

brings only minor changes in their optical spectra. ${ }^{84}$ Two main reasons are responsible for such a situation: partial overlap of the bands of both neutral and reduced forms, and low oscillator strength of the emerging new transitions. This appears to be the case for the molecules investigated. As indicated by TDDFT analysis (Table S4, ESI $\dagger$ ), the HOMO to SOMO transition is mainly responsible for a weak band at lower energies, whereas electronic excitation from SOMO to LUMO+2 explains the higher energy, more intense band. TDDFT simulation predicts one more strong transition (SOMO to LUMO) positioned in the NIR region. This signal, however, was not detected down to $1600 \mathrm{~nm}$, placing the SOMO-LUMO upper energy spacing limit at $c a$. $0.77 \mathrm{eV}$.
An intense 1:2:1 triplet EPR line was recorded during the electrochemical reduction of all the molecules, corroborating the emergence of a radical anion (Fig. 5 and Fig. S4, ESI $\dagger$ ). Despite notable differences in the chemical structure, the donor-to-acceptor ratio in particular, radical anions of all the molecules investigated demonstrate an identical triplet hyperfine splitting pattern. Best fitting a simulation model, involving hydrogen and nitrogen atoms, to the experimental spectra yields information about the number of nuclei and intensity of their magnetic interactions with the unpaired electron. For all six compounds it turns out to be enough to consider spin interaction with just two protons and all the 
Table 3 Properties of radical anions of compounds (1) to (6), electrogenerated in $0.1 \mathrm{M} \mathrm{Bu}_{4} \mathrm{NPF}_{6}$ in tetrahydrofuran

\begin{tabular}{|c|c|c|c|c|}
\hline \multirow[b]{2}{*}{ Compound } & \multirow{2}{*}{$\frac{\text { Absorption bands }[\mathrm{nm}]}{\text { In reduced state }}$} & \multicolumn{3}{|c|}{ EPR spectra } \\
\hline & & $g$-Factor & Nuclei $^{a}$ & $\operatorname{Hfcc}^{b}[\mathrm{G}]$ \\
\hline \multirow[t]{2}{*}{$(1)^{\bullet-}$} & \multirow[t]{2}{*}{$257 ; 338 ; 526$} & \multirow[t]{2}{*}{2.00558} & $\mathrm{H}(2)$ & 1.91 \\
\hline & & & $\mathrm{N}(2)$ & 0.08 \\
\hline \multirow[t]{2}{*}{$(2)^{\bullet-}$} & \multirow[t]{2}{*}{$259 ; 342 ; 362 ; 503$} & \multirow[t]{2}{*}{2.00558} & $\mathrm{H}(2)$ & 1.93 \\
\hline & & & $\mathrm{N}(2)$ & 0.11 \\
\hline \multirow[t]{2}{*}{$(3)^{\bullet-}$} & \multirow[t]{2}{*}{$259 ; 341 ; 372 ; 500$} & \multirow[t]{2}{*}{2.00555} & $\mathrm{H}(2)$ & 1.96 \\
\hline & & & $\mathrm{N}(2)$ & 0.05 \\
\hline \multirow[t]{2}{*}{$(4)^{\bullet-}$} & \multirow[t]{2}{*}{$264 ; 327 ; 447$} & \multirow[t]{2}{*}{2.00554} & $\mathrm{H}(2)$ & 1.96 \\
\hline & & & $\mathrm{N}(2)$ & 0.10 \\
\hline \multirow[t]{3}{*}{$(5)^{\bullet-}$} & \multirow[t]{3}{*}{$265 ; 336 ; 450$} & \multirow[t]{3}{*}{2.00557} & $\mathrm{H}(2)$ & 1.96 \\
\hline & & & $\mathrm{N}(2)$ & 0.11 \\
\hline & & & $\mathrm{N}(2)$ & 0.03 \\
\hline \multirow[t]{3}{*}{$(6)^{\bullet-}$} & \multirow[t]{3}{*}{$260 ; 330 ; 458$} & \multirow[t]{3}{*}{2.00558} & $\mathrm{H}(2)$ & 1.94 \\
\hline & & & $\mathrm{N}(2)$ & 0.12 \\
\hline & & & $\mathrm{N}(2)$ & 0.11 \\
\hline
\end{tabular}

${ }^{a}$ Values in parenthesis indicate the number of each nuclei included in a simulation model. ${ }^{b}$ Hyperfine coupling constant best-fit to an experimental spectrum.

nitrogens to accurately reproduce the experimental spectrum. Hyperfine coupling constants come in pairs affording a simple EPR lineshape indicative of a symmetric geometry of the delocalization space of the radical. This, and the identical $g$-factor values across all the compounds studied, suggest that the radical anion is predominantly localized around the 1,3,4oxadiazole acceptor units. Strikingly, the hyperfine coupling constants of nitrogen are very low compared to those of hydrogen nuclei, indicating a much higher spin density at the $\mathrm{C}^{3}$ carbon atom of the thiophene units neighbouring 1,3,4oxadiazole(s) than at the nitrogen atoms of the diazole moiety itself. Surprising as they might be, all the experimental observations are neatly vindicated by TDDFT computed radical anion spin densities (Fig. 5 and Fig. S4, ESI $\dagger$ ). High spin densities are predicted to prevail at the 1,3,4-oxadiazole moieties, albeit only at the carbon and oxygen atoms. For mono oxadiazole structures, an equal residual spin density is expected at each nitrogen atom, and for bioxadiazole ones, two groups of nitrogen atoms differing in spin density are identified in line with the EPR line simulation results. A remarkably low spin density is computed for the hydrogen bonded carbons of the phenylene linker in (5), explaining the reasons for the spectral pattern of this radical anion being identical with that of other anions. The only discrepancy is the computational prediction of spin density extending further down the oligothiophene arms, rather than being confined to the first thiophene unit away from the oxadiazole core; however, the TDDFT method is known to overestimate the extent of the delocalization of $\pi$-electrons.

\subsection{Electrochemistry of electrogenerated polymers}

Electrochemical oxidation of all six compounds investigated yields radical cation products with reactive $\alpha$-carbon unsubstituted thienyl groups (vide supra). Irreversible oxidation of the monomers generates radical cations, which readily couple with one another, giving oligomers and eventually polymeric films. From the second CV cycle onwards, well-defined polymer redox peaks accompanied by gradual increase in their current intensity were observed due to the formation of an electroactive layer. Repetitive oxidation using cyclic voltammetry delivers electroactive polymer deposits at the surface of the working electrode,${ }^{85}$ as shown in Fig. S2 (ESI $\dagger$ ). As opposed to oligothiophenes like quinquethiophene, or longer oligomers, which dimerize negligibly, ${ }^{74}$ polymerization of penta-(4), hexa-(6) and septa-cycle (2), (3), (5) compounds already runs effortlessly at their first oxidation potential. ${ }^{75,86}$ Only (1) has a quasi-reversible first oxidation peak, but its electroactive layer develops upon potential cycling past the second oxidation peak. All the electropolymerization products can be considered as alternating donor-acceptor copolymers of oligothiophenes and mono- and bi-oxadiazoles (plain or phenylene bridged) as depicted in Scheme S1 (ESI $\dagger$ ).

The obtained polymer layers are reversibly electroactive in a wide potential window (Fig. 6a and Fig. S5, ESI $\dagger$ ). In the p-doping process, the number of distinguishable redox systems depends on the donor-to-acceptor ratio, whereas for n-doping, only one redox system is seen. The onset oxidation potential of the polymer film decreases from poly(6) to poly(1) with the increasing ratio of donor to acceptor repeating units, and that trend is only broken at poly(2), whose oxidation potential is higher than of poly(3) (Table 4). Poly(2), having more alkyl groups, would be expected to oxidize lower than poly(3); alas, steric hindrance of the additional alkyl chains makes its oligoalkylthiophene repeating unit more twisted, disrupting conjugation along the macromolecule and elevating its oxidation potential. ${ }^{87,88}$ Upon polymerization, oligoalkylthiophene arms couple together, giving units doubled in size. The longer the oligothiophene arm, the greater the donor repeating unit in the polymer, which is why the oxidation potential difference between the monomer and its polymer gets bigger on going from poly $(\mathbf{6})(-0.36 \mathrm{~V})$ to poly $(\mathbf{1})(-0.55 \mathrm{~V})$ (Table S5, ESI $\dagger$ ). On top of that, an assortment of alkyl substitution patterns of the resulting polymers is possible (Scheme S1, ESI $\dagger$ ) and the shape of the oxidative branch of the $\mathrm{CV}$ of the polymers appears to be related to this structural feature. At the onset of oxidation, a sharp current growth takes place in polymers with alkyl-free mono- $(\operatorname{poly}(2))$, or bi-thiophene (poly(4), poly(5) and poly(6)) segments, whereas those with quater- (poly(3)) and sexithiophene (poly(1)) units enjoy a relatively smooth current ascent at the initial stages of doping. A high oxidation current of a conjugated polymer layer is often caused by substantial capacitive currents associated with counterion movement into the film. A high density of alkyl chains, on the one hand, makes the polymer layer less compact, while, on the other hand, it could obstruct the movement of ions within it. Longer plain oligothiophene segments exhibit a stacking tendency, giving aggregates which oxidize more easily, but at the same time are less permeable to ion passage. As a result, oxidation of such films starts at lower potentials and their doping current trace spreads out on the potential scale.

All the polymers investigated are stable towards reduction in anhydrous environments, indicative of their n-doping reversibility. Contrary to observations made for monomer molecules (vide supra), the elongation of the donor segment length hinders 
a)

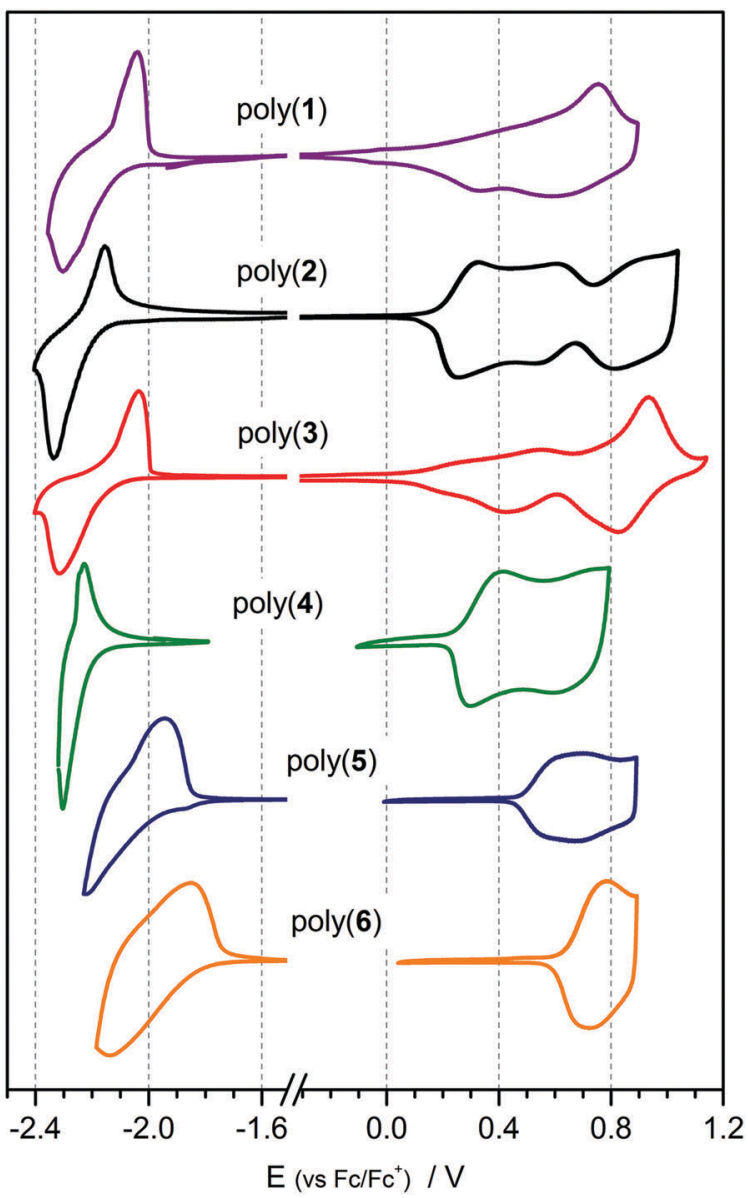

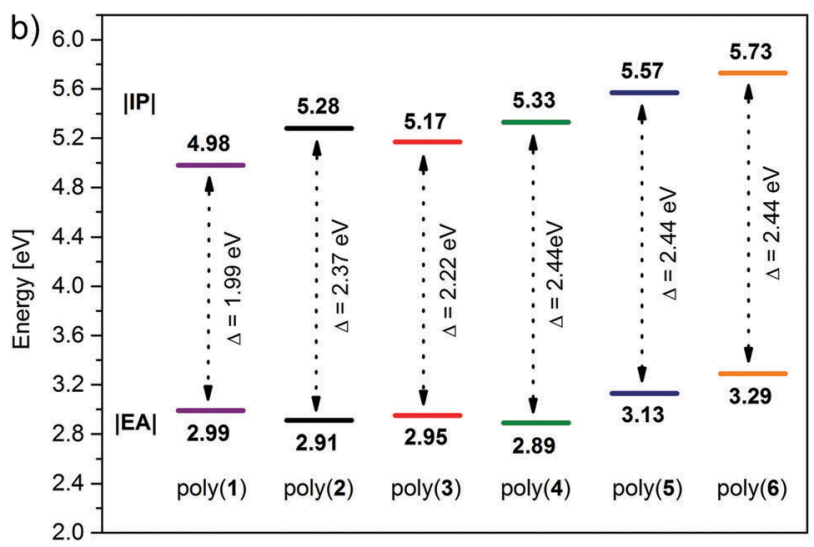

Fig. 6 (a) Cyclic voltammogram of the investigated polymers registered in $0.1 \mathrm{M} \mathrm{Bu}_{4} \mathrm{NPF}_{6}$ in dichloromethane for the anodic potential range and in tetrahydrofuran for the cathodic potential range. Scan rate: $100 \mathrm{mV} \mathrm{s}^{-1}$; (b) absolute values of the Ionization Potential (IP) and Electron Affinity (EA) of polymerization products.

electron uptake in $\operatorname{poly}(\mathbf{1}), \operatorname{poly}(\mathbf{2})$ and poly(3), making their reduction potentials less negative compared with that of their monomers. The reduction potentials of poly(4) and poly(5) are almost identical with those of their monomers, and only poly $(\mathbf{6})$ features a lower reduction potential than its monomer. The differences above are notably smaller in magnitude than the corresponding differences for the oxidation potential parameters
Table 4 Electrochemical properties of the investigated polymerization products determined from the redox data

\begin{tabular}{lllllll}
\hline Polymer & $E_{(\mathrm{onset})}^{\mathrm{ox}}[\mathrm{V}]$ & $E_{(\mathrm{onset})}^{\mathrm{red}}[\mathrm{V}]$ & $E^{\mathrm{ox} a}[\mathrm{~V}]$ & $|\mathrm{IP}|^{b}$ & $|\mathrm{EA}|^{c}$ & $E_{\mathrm{g}}^{\mathrm{el}}[\mathrm{eV}]$ \\
\hline Poly(1) & -0.12 & -2.11 & $-0.01 ;+0.52 ;+0.75$ & 4.98 & 2.99 & 1.99 \\
Poly(2) & +0.18 & -2.19 & $+0.34 ;+0.64 ;+0.89$ & 5.28 & 2.91 & 2.37 \\
Poly(3) & +0.07 & -2.15 & $+0.26 ;+0.56 ;+0.93$ & 5.17 & 2.95 & 2.22 \\
Poly(4) & +0.23 & -2.21 & $+0.41 ;+0.73$ & 5.33 & 2.89 & 2.44 \\
Poly(5) & +0.47 & -1.97 & +0.76 & 5.57 & 3.13 & 2.44 \\
Poly(6) & +0.63 & -1.81 & +0.78 & 5.73 & 3.29 & 2.44
\end{tabular}

${ }^{a}$ Peak of oxidation of the investigated $\pi$-conjugated layers. ${ }^{b}$ Calculated

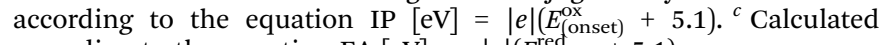
according to the equation $\mathrm{EA}[\mathrm{eV}]=-|e|\left(E_{\text {(onset) }}^{\text {red }}+5.1\right)$.

(Table S5, ESI $\dagger$ ), leading to an asymmetric band gap narrowing on going from monomers to polymers. A similar effect has been observed before for oligothiophene derivatives of other 3,4-diazole electron-withdrawing units - triazole and thiadiazole. . $^{890}$ Reconfiguration of the frontier molecular orbitals in the polymer impacts the characteristic transition energies between them, translating to distinctive absorptive features of these materials in the visible light range.

\section{4. p-Doping process of polymers}

UV-Vis-NIR and EPR spectroelectrochemistries are a powerful combination of complimentary analytical tools for elucidating the processes of generation and annihilation of charge carriers in semiconducting polymers. Commencing investigations at the neutral (undoped) state, it is instructive to analyse the UV-Vis-NIR spectra of the polymers. Absorption maxima as well as their onsets deliver important and comparative information about the energies of electron transitions in the undoped state. Extrapolation of the low energy tail of the $\pi-\pi^{*}$ absorption peak (Fig. 7) gives the optical band gap, which differs from electrochemical one by the electron-hole binding energy (Table 5). ${ }^{91}$ For the polymers investigated, the optical band-gap is between $1.93 \mathrm{eV}$ for poly(1) and $2.24 \mathrm{eV}$ for poly(5), and is lower compared with the electrochemical one because of the effect described above. These band gap values position themselves in the visible light range and their variation translates into a distinctive colouration of each polymer. ${ }^{92} \operatorname{Poly}(\mathbf{1})$, poly(2) and poly(3) share similar pink-red tones, poly(4) is orange-pink, whereas poly(5) and poly(6) are amber coloured. The absorption peak of two polymers - $\operatorname{poly}(2)$ and poly(4) - reveals an interesting vibronic structure, indicative of the strong coupling of electron and vibrational modes in ordered polymer layers. ${ }^{29}$ Interestingly, these two polymers feature the highest alkyl chain grafting density, taken as the number of decylthiophene groups with respect to the total number of $\pi$-conjugated rings in a repeating unit (Scheme S1, ESI $\dagger$ ), being 4 out of $7(0.57)$ in poly(2), and 2 out of 5 (0.4) in poly(4). Beside alkyl chain density, the alkane chain length itself is an important factor responsible for this ordering effect, since long alkyl chains are known to promote cooperative self-aggregation, particularly in higher molecular weight structures with restricted rotation opportunities. Curiously enough, intramolecular hydrogen-oxygen interactions fail to deliver an equally strong self-rigidification 

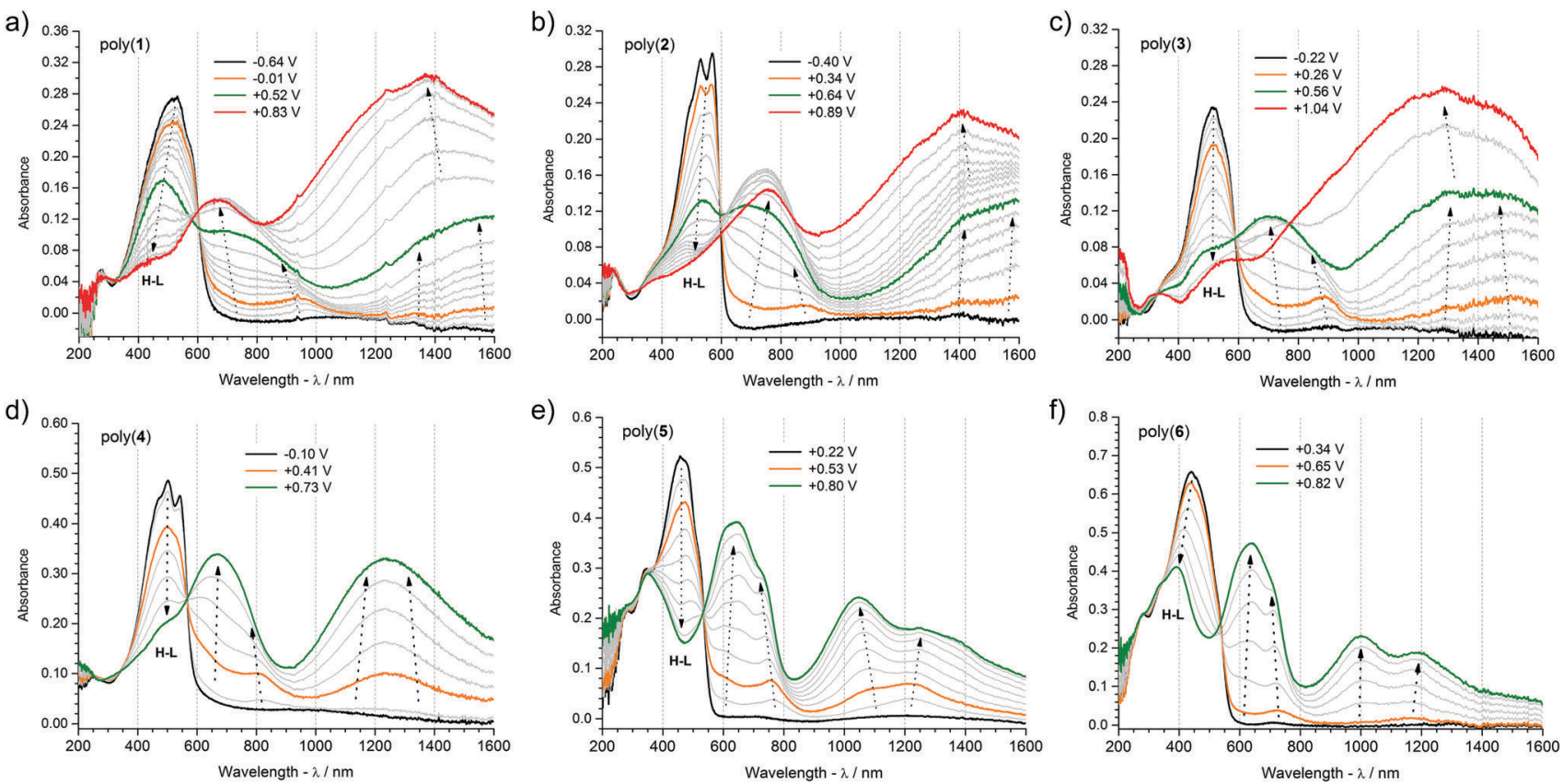

Fig. 7 UV-Vis-NIR spectra of: (a) poly(1), (b) poly(2), (c) poly(3), (d) poly(4), (e) poly(5), (f) poly(6) as a function of the apparent working electrode potential applied during the $p$-doping process. Coloured lines correspond to: neutral state of the polymer (black line) and polymer doped to: polaron (orange line), polaron pairs (green line) and bipolaron (red line).

Table 5 Spectroscopic properties of polymerization products, including electron transitions assigned to different charge carriers: polarons, polaron-pairs and bipolarons

\begin{tabular}{llllll}
\hline Compound & $\lambda_{\max }^{a}$ & $E_{\mathrm{g}}^{\text {opt }}[\mathrm{eV}]$ & Polaron $^{b}$ & Polaron-pair $^{b}$ & Bipolaron $^{b}$ \\
\hline Poly(1) & 523 & 1.93 & $953 ; 1580$ & $686 ; 1418$ & 1374 \\
Poly(2) & $530 ; 573$ & 2.02 & $880 ; 1609$ & $748 ; 1427$ & 1405 \\
Poly(3) & 515 & 1.97 & $892 ; 1495$ & $702 ; 1344$ & 1297 \\
Poly(4) & $501 ; 544$ & 2.11 & $813 ; 1304$ & $673 ; 1166$ & - \\
Poly(5) & 464 & 2.24 & $763 ; 1226$ & $643 ; 1046$ & - \\
Poly(6) & 440 & 2.19 & $725 ; 1188$ & $637 ; 1001$ & -
\end{tabular}

${ }^{a}$ Absorption maxima in the neutral state of the polymerization products $[\mathrm{nm}] .{ }^{b}$ Optical transitions for the respective charge carriers [nm].

effect, which could be particularly expected in poly(5) where such non-covalent bonds can develop between 1,3,4-oxadiazole units and alkylthiophene and phenylene moieties. All in all, the observed vibronic signatures diminish upon the application of an electric potential to the polymer film. During the p-doping, charges injected into the polymer film induce geometric reorganization of the atomic lattice, while charge counterbalancing ions incorporate themselves between the doped chains, disrupting the supramolecular arrangement of the macromolecules.

Electrochemical doping coupled with in situ UV-Vis-NIR and Electron Paramagnetic Resonance spectroscopies delivers a unique opportunity to determine the type of charge carriers generated in this process. Cyclic voltammetry data provide accurate and reproducible information about the total doping charge consumed, EPR spectroscopy is selectively sensitive to any paramagnetic species present, and UV-Vis-NIR spectroscopy detects electron transitions taking place in a doped polymer film.
A comprehensive picture of the doping mechanism can be built when parameters like redox peak potentials, electron transition energies and concentrations of paramagnetic species (Fig. 7, 8 and Table 5) are analysed in parallel. Correlating the onset oxidation potentials with relative concentration of spins leads to the conclusion that in all the polymers investigated, the p-doping process begins with formation of paramagnetic charge carriers, so-called polarons. Further oxidation increases the number of paramagnetic species with a concomitant decrease of the EPR signal linewidth. Decreasing the EPR signal linewidth indicates a weakening of the dipolar interactions between polaronic spins that can be brought about by their intra- or intermolecular pairing. Hindering these, as has been neatly demonstrated in isolated planar polythiophene, delivers a sharp and constant signal linewidth EPR signal in a wide range of potentials, indicative of the undisturbed presence of polarons. ${ }^{93}$ For poly(1) to poly(3), whose onset oxidation potential is lowest and p-doping potential window is wider than of poly(4) to poly(6), pushing the doping process further brings about the transformation of paramagnetic charge carriers into diamagnetic ones. The EPR results show this clearly, when at the final stages of doping, the concentration of paramagnetic species decreases and the EPR signals broaden, indicative of the creation of spinless bipolarons which act as efficient mediators of dipolar interactions between the remaining polarons. The absence of this effect in poly(4) to poly(6) should be assigned to a better stabilization of the diamagnetic charge carriers on longer oligothiophene repeating unit segments. This phenomenon was observed previously for model oligothiophenes, where sexi- or octithiophene segments were able to stabilize dications. ${ }^{94,95}$ Further conclusions can be drawn when UV-Vis-NIR spectra are also considered. Inspecting the collections of UV-Vis-NIR spectra recorded at a steadily 

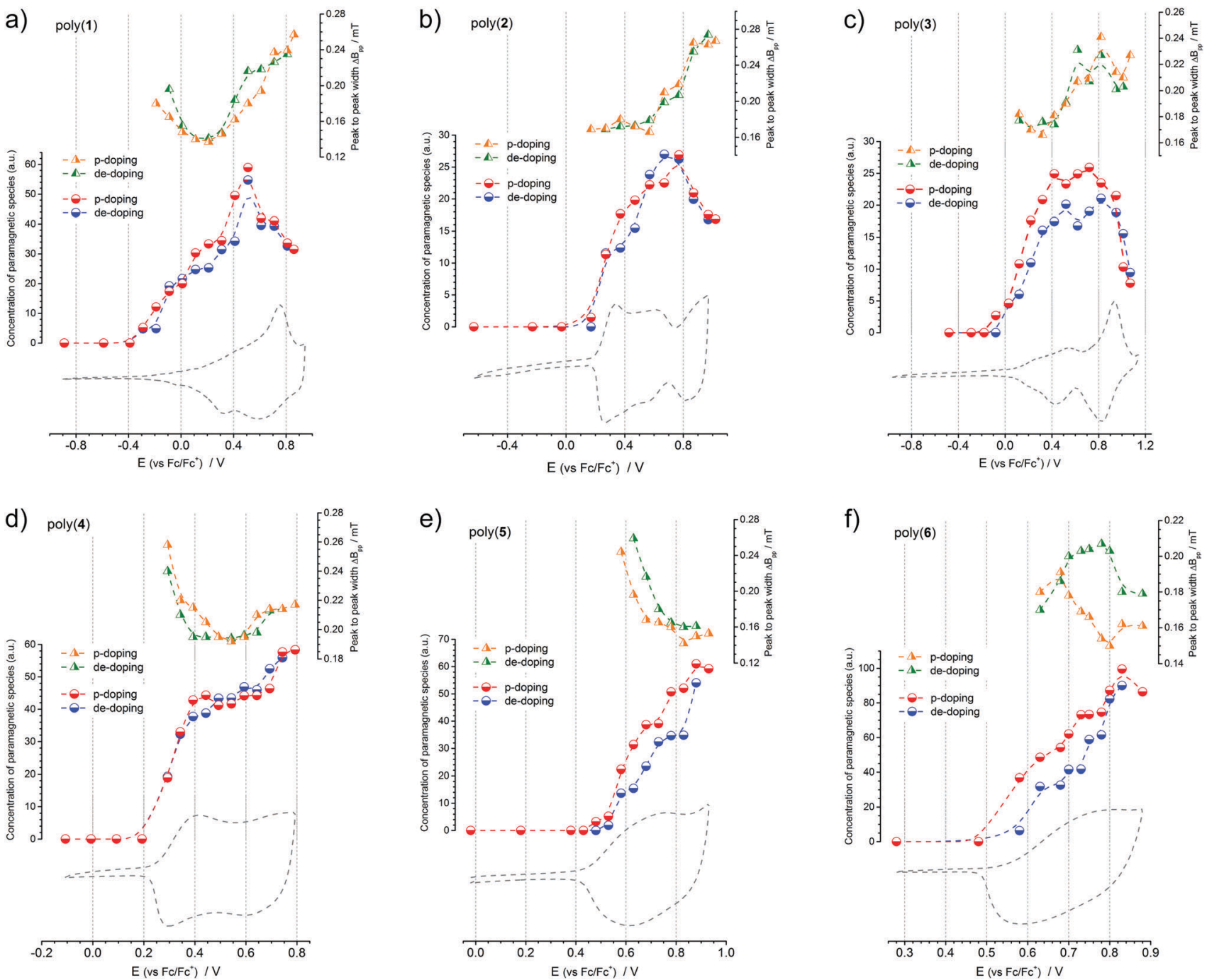

Fig. 8 Concentration of paramagnetic charge carriers with the linewidth of their EPR spectra $\left(\Delta B_{\mathrm{pp}}\right)$, and cyclic voltammetry trace of (a) poly(1), (b) poly(2), (c) poly(3), (d) poly(4), (e) poly(5), (f) poly(6), registered during the p-doping and de-doping process.

incremented doping potential, for each polymer layer, two spectral bands are observed growing simultaneously with the increasing potential. For poly(5) and poly(6), from the beginning of doping, each band consists of two absorption maxima, whereas for the other polymers only one maximum is seen, shifting towards higher energies as the doping proceeds. Clearly, paramagnetic charge carriers created on the polymer chains are responsible for these bands. However, the formation of other charge carriers cannot be excluded, especially the creation of polaron pairs. ${ }^{96}$ This would explain the presence of four maxima observed for poly(5) and poly(6) - since both these charge carriers are characterized by two allowed electron transitions. Shifting of polaron band in poly(1) to poly(4) can also be ascribed to its gradual transformation into a polaron pair band. Subsequent changes in the spectra can be seen for poly(1) to poly(3) at the higher doping potentials applied. A third band begins to dominate the spectrum after a certain potential, and its appearance is connected with the formation of bipolarons on the polymer chain.
Summarizing, three kinds of charge carriers are generated in the polymers investigated upon their p-doping process: polarons, polaron-pairs and bipolarons. For polymers with a higher number of electron-releasing thienyl units, that is poly(1) to poly(3), three peaks observed on the voltammograms correspond to the formation of polaron, polaron-pair and bipolaron species, respectively. For poly(4), the two peaks can be ascribed to the formation of polarons and polaron-pairs. As for poly(5) and poly(6), the polymers most abundant in electron-withdrawing moieties, both polarons and polaron-pairs are formed in parallel, which is why only one cyclic voltammetric peak is observed.

\section{Conclusions}

Synthesis, electrochemical and spectroelectrochemical studies of a series of donor-acceptor-donor (D-A-D) $\pi$-conjugated alkyl functionalized oligothiophenes incorporating central 
1,3,4-oxadiazole electron-withdrawing motifs are presented. Traceable, yet elaborate, relationships between their spectral parameters, like absorption and fluorescence maxima and luminescence quantum yield, are found to depend on both the ratio of acceptor to donor units in these molecules and also on the intramolecular structure-ordering interactions developing across their electron-withdrawing and electron-releasing fragments. As for typical D-A-D molecules, the compounds investigated exhibit electroactivity in both oxidation and reduction processes. As regards the oxidation process, a clear trend of a decreasing oxidation potential with a growing contribution of electronreleasing groups was observed. For reduction, this dependence is not that obvious, since increasing the contribution of either the electron acceptor or electron donor units makes the reduction potential of the molecules less negative. All the compounds demonstrated a reversible first reduction step, followed by a quasi-reversible second one, whereas the oxidation of compounds (2) to (6) was irreversible, leading to formation of electroactive polymers. Compound (1) electro-polymerized as well, but only at potentials above its second oxidation peak. The obtained polymers demonstrated different colours, depending on their repeating unit structure. All the materials demonstrated different electrochromic properties, some featuring multielectrochromic traits. Using UV-Vis-NIR spectroelectrochemistry and EPR spectroelectrochemistry, different charge carriers were identified in polymer films upon their electrochemical doping. These were: polarons, polaron pairs or bipolarons. It was observed that for polymers with relatively short electron-releasing segments (quaterthiophene), polarons and polaron-pairs are the only possible charge carriers. Only polymers with longer oligoalkylthiophene segments, like sexi- or octithiophene, could stabilize diamagnetic bipolarons. This leads to a conclusion that the electronwithdrawing oxadiazole moiety acts as a conjugation break for positive charge carriers delocalized on donor fragments of the polymer chain, leading to a suppressed electronic communication between the electron-releasing oligothiophene fragments.

\section{Conflicts of interest}

The authors declare no conflicts of interest.

\section{Acknowledgements}

Financial support of the Polish National Science Centre grant no. 2011/03/D/ST5/06042 is gratefully acknowledged. Quantum chemical computations were supported by Pl-Grid infrastructure. A. Kostyuchenko acknowledges partial financial support from the Russian Foundation for Basic Research (15-43-04313a) and the Ministry of Education and Science of the Russian Federation (agreement number 02.a03.21.0008). T. Zheleznova, K. Andryukhova and A. Fisyuk acknowledge the support of the Ministry of Education and Science of the Russian Federation, project number 4.1657.2017/4.6. A. Pron acknowledges partial financial support of the Warsaw University of Technology.

\section{References}

1 X. Guo, M. Baumgarten and K. Müllen, Prog. Polym. Sci., 2013, 38, 1832-1908.

2 K.-J. Baeg, M. Caironi and Y.-Y. Noh, Adv. Mater., 2013, 25, 4210-4244.

3 P. Bujak, I. Kulszewicz-Bajer, M. Zagorska, V. Maurel, I. Wielgus and A. Pron, Chem. Soc. Rev., 2013, 42, 8895.

4 F. Carpi and D. De Rossi, Opt. Laser Technol., 2006, 38, 292-305.

5 H. Klauk, Chem. Soc. Rev., 2010, 39, 2643.

6 J. Wang, F. Zhang, J. Zhang, W. Tang, A. Tang, H. Peng, Z. Xu, F. Teng and Y. Wang, J. Photochem. Photobiol., C, 2013, 17, 69-104.

7 M. G. Murali, P. Naveen, D. Udayakumar, V. Yadav and R. Srivastava, Tetrahedron Lett., 2012, 53, 157-161.

8 R. Liu, X. Xu, J. Peng, C. Yao, J. Wang and L. Li, RSC Adv., 2015, 5, 36568-36574.

9 S. K. Pathak, R. K. Gupta, S. Nath, D. S. S. Rao, S. K. Prasad and A. S. Achalkumar, J. Mater. Chem. C, 2015, 3, 2940-2952.

10 L. Bian, E. Zhu, J. Tang, W. Tang and F. Zhang, Prog. Polym. Sci., 2012, 37, 1292-1331.

11 X. Zhan and D. Zhu, Polym. Chem., 2010, 1, 409.

12 A. Facchetti, Chem. Mater., 2011, 23, 733-758.

13 P. M. Beaujuge and J. R. Reynolds, Chem. Rev., 2010, 110, 268-320.

14 G. Gunbas and L. Toppare, Chem. Commun., 2012, 48, 1083-1101.

15 R. J. Mortimer, A. L. Dyer and J. R. Reynolds, Displays, 2006, 27, 2-18.

16 H. Sirringhaus, Adv. Mater., 2014, 26, 1319-1335.

17 J. Mei, Y. Diao, A. L. Appleton, L. Fang and Z. Bao, J. Am. Chem. Soc., 2013, 135, 6724-6746.

18 M. I. Özkut, M. P. Algi, Z. Öztaş, F. Algi, A. M. Önal and A. Cihaner, Macromolecules, 2012, 45, 729-734.

19 O. Gidron, N. Varsano, L. J. W. Shimon, G. Leitus and M. Bendikov, Chem. Commun., 2013, 49, 6256.

20 J. Lee, K. Shizu, H. Tanaka, H. Nomura, T. Yasuda and C. Adachi, J. Mater. Chem. C, 2013, 1, 4599.

21 S. Seo, H. Shin, C. Park, H. Lim and E. Kim, Macromol. Res., 2013, 21, 284-289.

22 N. L. Bill, J. M. Lim, C. M. Davis, S. Bähring, J. O. Jeppesen, D. Kim and J. L. Sessler, Chem. Commun., 2014, 50, 6758.

23 U. Mitschke and P. Bäuerle, J. Mater. Chem., 2000, 10, 1471-1507.

24 K. Takimiya, I. Osaka and M. Nakano, Chem. Mater., 2014, 26, 587-593.

25 S. Tarkuc, Y. A. Udum and L. Toppare, Thin Solid Films, 2012, 520, 2960-2965.

26 J. Chen and Y. Cao, Acc. Chem. Res., 2009, 42, 1709-1718.

27 P. Camurlu, C. Gültekin and Z. Bicil, Electrochim. Acta, 2012, 61, 50-56.

28 M. D. Levi, A. S. Fisyuk, R. Demadrille, E. Markevich, Y. Gofer, D. Aurbach and A. Pron, Chem. Commun., 2006, 3299.

29 J. Roncali, Macromol. Rapid Commun., 2007, 28, 1761-1775. 
30 Y. Wu and W. Zhu, Chem. Soc. Rev., 2013, 42, 2039-2058.

31 K. Kotwica, A. S. Kostyuchenko, P. Data, T. Marszalek, L. Skorka, T. Jaroch, S. Kacka, M. Zagorska, R. Nowakowski, A. P. Monkman, A. S. Fisyuk, W. Pisula and A. Pron, Chem. Eur. J., 2016, 22, 11795-11806.

32 Z. Pomerantz, M. D. Levi, G. Salitra, R. Demadrille, A. Fisyuk, A. Zaban, D. Aurbach and A. Pron, Phys. Chem. Chem. Phys., 2008, 10, 1032-1042.

33 F. Demanze, a Yassar and F. Garnier, Macromolecules, 1996, 29, 4267-4273.

34 A. V. Akkuratov, D. K. Susarova, O. A. Mukhacheva and P. A. Troshin, Mendeleev Commun., 2016, 26, 248-250.

35 C. B. Nielsen and I. McCulloch, Prog. Polym. Sci., 2013, 38, 2053-2069.

36 A. Kurowska, A. S. Kostyuchenko, P. Zassowski, L. Skorka, V. L. Yurpalov, A. S. Fisyuk, A. Pron and W. Domagala, J. Phys. Chem. C, 2014, 118, 25176-25189.

37 S. I. Panchamukhi, N. Belavagi, M. H. Rabinal and I. A. Khazi, J. Fluoresc., 2011, 21, 1515-1519.

38 F. Chen, T. Tian, B. Bai, J. Wang, H. Wang and M. Li, J. Mater. Chem. C, 2016, 4, 4451-4458.

39 A. S. Kostyuchenko, G. Wiosna-Salyga, A. Kurowska, M. Zagorska, B. Luszczynska, R. Grykien, I. Glowacki, A. S. Fisyuk, W. Domagala and A. Pron, J. Mater. Sci., 2016, 51, 1-13.

40 N. E. Jackson, B. M. Savoie, K. L. Kohlstedt, M. Olvera de la Cruz, G. C. Schatz, L. X. Chen and M. A. Ratner, J. Am. Chem. Soc., 2013, 135, 10475-10483.

41 G. Conboy, H. J. Spencer, E. Angioni, A. L. Kanibolotsky, N. J. Findlay, S. J. Coles, C. Wilson, M. B. Pitak, C. Risko, V. Coropceanu, J.-L. Brédas and P. J. Skabara, Mater. Horiz., 2016, 3, 333-339.

42 A. S. Fisyuk, R. Demadrille, C. Querner, M. Zagorska, J. Bleuse and A. Pron, New J. Chem., 2005, 29, 707.

43 A. S. Kostyuchenko, A. M. Averkov and A. S. Fisyuk, Org. Lett., 2014, 16, 1833-1835.

44 A. S. Kostyuchenko, V. L. Yurpalov, A. Kurowska, W. Domagala, A. Pron and A. S. Fisyuk, Beilstein J. Org. Chem., 2014, 10, 1596-1602.

45 A. S. Kostyuchenko, E. A. Drozdova and A. S. Fisyuk, Chem. Heterocycl. Compd., 2017, 53, 92-96.

46 A. S. Kostyuchenko, T. Y. Zheleznova, A. J. Stasyuk, A. Kurowska, W. Domagala, A. Pron and A. S. Fisyuk, Beilstein J. Org. Chem., 2017, 13, 313-322.

47 K. Kotwica, E. Kurach, G. Louarn, A. S. Kostyuchenko, A. S. Fisyuk, M. Zagorska and A. Pron, Electrochim. Acta, 2013, 111, 491-498.

48 J. A. Joule and K. Mills, Heterocyclic Chemistry, Wiley, 5th edn, 2010.

49 W. R. Dawson and M. W. Windsor, J. Phys. Chem., 1968, 72, 3251-3260.

50 G. Weber and F. W. J. Teale, Trans. Faraday Soc., 1957, 53, 646.

51 M. W. Allen, Thermo Sci., 2010, 1-4.

52 D. R. Duling, J. Magn. Reson., Ser. B, 1994, 104, 105-110.

53 C. Lee, W. Yang and R. G. Parr, Phys. Rev. B: Condens. Matter Mater. Phys., 1988, 37, 785-789.
54 A. D. Becke, J. Chem. Phys., 1993, 98, 1372.

55 A. Becke, J. Chem. Phys., 1993, 98, 5648-5652.

56 T. Yanai, D. P. Tew and N. C. Handy, Chem. Phys. Lett., 2004, 393, 51-57.

57 A.-R. Allouche, J. Comput. Chem., 2011, 32, 174-182.

58 M. J. Frisch, G. W. Trucks, H. B. Schlegel, G. E. Scuseria, M. A. Robb, J. R. Cheeseman, G. Scalmani, V. Barone, B. Mennucci, G. A. Petersson, H. Nakatsuji, M. Caricato, X. Li, H. P. Hratchian, A. F. Izmaylov, J. Bloino, G. Zheng, J. L. Sonnenberg, M. Hada, M. Ehara, K. Toyota, R. Fukuda, J. Hasegawa, M. Ishida, T. Nakajima, Y. Honda, O. Kitao, H. Nakai, T. Vreven, J. A. Montgomery, Jr., J. E. Peralta, F. Ogliaro, M. Bearpark, J. J. Heyd, E. Brothers, K. N. Kudin, V. N. Staroverov, R. Kobayashi, J. Normand, K. Raghavachari, A. Rendell, J. C. Burant, S. S. Iyengar, J. Tomasi, M. Cossi, N. Rega, J. M. Millam, M. Klene, J. E. Knox, J. B. Cross, V. Bakken, C. Adamo, J. Jaramillo, R. Gomperts, R. E. Stratmann, O. Yazyev, A. J. Austin, R. Cammi, C. Pomelli, J. W. Ochterski, R. L. Martin, K. Morokuma, V. G. Zakrzewski, G. A. Voth, P. Salvador, J. J. Dannenberg, S. Dapprich, A. D. Daniels, Ö. Farkas, J. B. Foresman, J. V. Ortiz, J. Cioslowski and D. J. Fox, Gaussian 09, Revision B.01, Gaussian, Inc., Wallingford CT, 2009.

59 E. Fabiano, F. Della Sala and R. Cingolani, Physica Status Solidi C: Conferences, American Chemical Society, 2004, vol. 1, pp. 539-542.

60 R. A. J. Janssen, L. Smilowitz, N. S. Sariciftci and D. Moses, J. Chem. Phys., 1994, 101, 1787.

61 U. Mitschke, T. Debaerdemaeker and P. Bäuerle, Eur. J. Org. Chem., 2000, 425-437.

62 S. Qu, X. Chen, X. Shao, F. Li, H. Zhang, H. Wang, P. Zhang, Z. Yu, K. Wu, Y. Wang and M. Li, J. Mater. Chem., 2008, 18, 3954.

63 R. Rybakiewicz, P. Gawrys, D. Tsikritzis, K. Emmanouil, S. Kennou, M. Zagorska and A. Pron, Electrochim. Acta, 2013, 96, 13-17.

64 P. S. Johnson, I. Boukahil, F. J. Himpsel, K. L. Kearns, J. H. Kang, J.-C. Lin, A. Leugers, G. Meyers, S. Mukhopadhyay, D. H. K. Jackson and T. F. Kuech, J. Phys. Chem. C, 2016, 120, 1366-1374.

65 P. I. Djurovich, E. I. Mayo, S. R. Forrest and M. E. Thompson, Org. Electron., 2009, 10, 515-520.

66 C. Arantes, M. Scholz, R. Schmidt, V. Dehm, M. L. M. Rocco, A. Schöll, F. Reinert and F. Würthner, Appl. Phys. A: Mater. Sci. Process., 2012, 108, 629-637.

67 J. Sworakowski, J. Lipiński and K. Janus, Org. Electron., 2016, 33, 300-310.

68 B. Nessakh, G. Horowitz, F. Garnier, F. Deloffre, P. Srivastava and A. Yassar, J. Electroanal. Chem., 1995, 399, 97-103.

69 M. Lapkowski, M. Kolodziej-Sadlok, J. Zak, S. Guillerez and G. Bidan, Adv. Mater., 2001, 13, 803-806.

70 W. Domagala, M. Lapkowski, S. Guillerez and G. Bidan, Electrochim. Acta, 2003, 48, 2379-2388.

71 M. Łapkowski, J. Zak, M. Kolodziej-Sadlok, S. Guillerez and G. Bidan, Electrochim. Acta, 2001, 46, 4001-4006. 
72 J. Zak, M. Lapkowski, S. Guillerez and G. Bidan, J. Solid State Electrochem., 2006, 10, 134-139.

73 K. Meerholz and J. Heinze, Electrochim. Acta, 1996, 41, 1839-1854.

74 M. Barth, S. Guilerez, G. Bidan, G. Bras and M. Łapkowski, Electrochim. Acta, 2000, 45, 4409-4417.

75 J. Guay, P. Kasai, A. Diaz, R. Wu, J. M. Tour and L. H. Dao, Chem. Mater., 1992, 4, 1097-1105.

76 P. T. Henderson and D. M. Collard, Chem. Mater., 1995, 7, 1879-1889.

77 P. Bäuerle, U. Segelbacher, K.-U. Gaudl, D. Huttenlocher and M. Mehring, Angew. Chem., Int. Ed. Engl., 1993, 32, 76-78.

78 T. M. McCormick, C. R. Bridges, E. I. Carrera, P. M. Dicarmine, G. L. Gibson, J. Hollinger, L. M. Kozycz and D. S. Seferos, Macromolecules, 2013, 46, 3879-3886.

79 H. Phillips, Z. Zheng, E. Geva and B. D. Dunietz, Org. Electron., 2014, 15, 1509-1520.

80 S. Pluczyk, P. Zassowski, R. Rybakiewicz, R. Wielgosz, M. Zagorska, M. Lapkowski and A. Pron, RSC Adv., 2015, 5, 7401-7412.

81 S. Pluczyk, P. Zassowski, M. Lapkowski, E. SchabBalcerzak and M. Grucela, J. Electroanal. Chem., 2015, 745, 14-21.

82 P. Data, A. Kurowska, S. Pluczyk, P. Zassowski, P. Pander, R. Jedrysiak, M. Czwartosz, L. Otulakowski, J. Suwinski, M. Lapkowski and A. P. Monkman, J. Phys. Chem. C, 2016, 120, 2070-2078.
83 M. Gora, S. Pluczyk, P. Zassowski, W. Krzywiec, M. Zagorska, J. Mieczkowski, M. Lapkowski and A. Pron, Synth. Met., 2016, 216, 75-82.

84 S. Pluczyk, P. Zassowski, C. Quinton, P. Audebert, V. Alain-Rizzo and M. Lapkowski, J. Phys. Chem. C, 2016, 120, 4382-4391.

85 C. A. Thomas, K. Zong, K. A. Abboud, P. J. Steel and J. R. Reynolds, J. Am. Chem. Soc., 2004, 126, 16440-16450.

86 P. Audebert, J.-M. Catel, G. Le Coustumer, V. Duchenet and P. Hapiot, J. Phys. Chem. B, 1998, 102, 8661-8669.

87 S. S. Zade and M. Bendikov, Chem. - Eur. J., 2007, 13, 3688-3700. 88 I. Osaka and K. Takimiya, Polymer, 2015, 59, A1-A15.

89 H. a M. Van Mullekom, J. a J. M. Vekemans, E. E. Havinga and E. W. Meijer, Developments in the chemistry and band gap engineering of donor-acceptor substituted conjugated polymers, 2001, vol. 32 .

90 M. D. Levi and D. Aurbach, J. Power Sources, 2008, 180, 902-908.

91 J.-L. Bredas, Mater. Horiz., 2014, 1, 17-19.

92 P. Monk, R. Mortimer and D. Rosseinsky, Electrochromism and Electrochromic Devices, Cambridge University Press, Cambridge, 2007.

93 R. Shomura, K. Sugiyasu, T. Yasuda, A. Sato and M. Takeuchi, Macromolecules, 2012, 45, 3759-3771.

94 M. G. Hill, J. F. Penneau, B. Zinger, K. R. Mann and L. L. Miller, Chem. Mater., 1992, 4, 1106-1113.

95 Y. Hong, Y. Yu and L. L. Miller, Synth. Met., 1995, 74, 133-135. 96 J. W. Jang, K. W. Lee and C. E. Lee, Solid State Commun., 2004, 131, 697-700. 